\title{
Nanoparticle analysis for blood flow of Prandtl fluid model with stenosis
}

\author{
Sohail Nadeem ${ }^{*}$, Shagufta ljaz ${ }^{1}$ and Noreen Sher Akbar ${ }^{2}$
}

\begin{abstract}
In this article, we have discussed blood flow in the nano-Prandtl fluid flow analysis in tapered stenosed arteries. The occurrence of nanoparticle fraction and heat transfer was found. Gravitational effects were also considered because the tube was taken vertically upward. Homotopy perturbation method was used to find the analytical solution of coupled nonlinear differential equations. Physical features have been discussed through graphs of concentration profile $\sigma$, velocity profile $w$, resistance impedance $\lambda$, temperature profile $\theta$, wall shear stress $S_{r z}$, wall shear stress at the stenosis throat $\tau_{s}$, and the stream lines.
\end{abstract}

Keywords: Prandtl fluid; Tapered artery; Nanoparticles; Blood flow; Stenosis; Homotopy perturbation method

\section{Background}

Blood flow in the artery has some important aspects due to engineering as well as medical application points of view. The hemodynamic behavior of the blood flow is influenced by the presence of arterial stenosis. If stenosis is present in the artery, normal blood flow is disturbed. Thurston and Chien et al. [1,2] present the viscoelastic properties of the blood. According to them, the arterial configuration is closely connected to blood flow. Arteries which are basically considered as living tissues need a supply of metabolites including oxygen and removal of waste products. Aroesty and Gross [3] have discussed the pulsatile flow of blood in the small blood vessels. Chaturani and Ponnalagar Samy [4] reported the theory of Aroesty and Gross [3] and studied the pulsatile flow of blood in stenosed arteries modeling blood as Casson fluid. Scott Blair and Spanner [5] discussed that blood as Casson fluid is valid for moderate shear rate, and the validity of Casson and Herschel-Bulkley theory for blood flow is the same. In another article, Siddiqui et al. [6] discussed Casson fluid in arterial stenosis. Pulsatile flow of blood for a modified second-grade fluid model is presented by Massoudi and Phuoc [7].

\footnotetext{
${ }^{*}$ Correspondence: snqau@hotmail.com

1 Department of Mathematics, Quaid-i-Azam University 45320, Islamabad 44000, Pakistan

Full list of author information is available at the end of the article
}

Mekheimer and El Kot [8] examined the micropolar fluid model for axisymmetric blood flow through an axially nonsymmetric but radially symmetric mild stenosis tapered artery. Mandal [9] presented unsteady flow analysis for blood by treating blood as a non-Newtonian fluid through tapered arteries with stenosis. He discussed the numerical solution for the flow equations. Varshney et al. [10] considered the generalized power law fluid model for blood flow in the artery considering multiple stenosis. They present a numerical study under the action of a transverse magnetic field. A power law fluid model for blood flow through a tapered artery with stenosis is recently developed by Nadeem et al. [11]. In another article, Nadeem and Akbar [12] revisited the flow analysis of Nadeem et al. [11] for Jeffrey fluid. Mustafa et al. [13] make the analysis of blood flow for the generalized Newtonian fluid through a couple of irregular arterial stenosis. Blood flow with an irregular stenosis in the presence of magnetic field has been touched by Abdullah et al. [14].

Nanofluids are the fluids of nanometer-sized particles of metals, oxides, carbides, nitrides, or nanotubes. Nowadays, nanofluids, among researchers, are considered an active area of research. In fact, nanofluids are a suspension of nanosized solid particles in a base fluid. The nanofluids have high thermal conductivity as compared to the base fluid. Nanofluids basically increase heat transfer rate. Recent articles on nanofluids have been cited [15-20].

The main theme of the present article is to discuss the nanofluid analysis for steady blood flow of the Prandtl

\section{Springer}

(c) 2013 Nadeem et al: licensee Springer. This is an Open Access article distributed under the terms of the Creative Commons Attribution License (http://creativecommons.org/licenses/by/2.0), which permits unrestricted use, distribution, and reproduction in any medium, provided the original work is properly cited. 
model with stenosis. To the best of the authors' knowledge, blood flow analysis for nanofluids has not been investigated so far. We arranged this article in the following manner. The 'Methods' section consists of mathematical formulation and the solution expressions for velocity, temperature, nanoparticle, resistance impedance, wall shear stress, and shearing stress at the stenosis throat. The 'Results and discussion' section analyzes the salient features of the problem by graphical illustration.

\section{Methods}

\section{Formulation of the problem}

Consider the flow of incompressible Prandtl fluid lying in a tube having the length $L$. We are considering the cylindrical coordinate system in such a way that $\tilde{u}, \tilde{v}$, and $\tilde{w}$ are the velocity components in $\bar{r}, \bar{\theta}$, and $\bar{z}$ directions. The governing equations of the steady incompressible Prandtl fluid are as follows [11]:

$$
\begin{aligned}
\frac{\partial \tilde{u}}{\partial \bar{r}}+\frac{\tilde{u}}{\bar{r}}+\frac{\partial \tilde{w}}{\partial \bar{z}}= & , \\
\rho\left(\tilde{u} \frac{\partial \tilde{u}}{\partial \bar{r}}+\tilde{w} \frac{\partial \tilde{u}}{\partial \bar{z}}\right)= & -\frac{\partial \bar{p}}{\partial \bar{r}}+\frac{1}{\bar{r}} \frac{\partial}{\partial \bar{r}}\left(\bar{r} S_{\bar{r} \bar{r}}\right)+\frac{\partial}{\partial \bar{z}}\left(\bar{r} S_{\bar{r} \bar{z})}\right)-\frac{S_{\bar{\theta} \bar{\theta}}}{\bar{r}} \\
\rho\left(\tilde{u} \frac{\partial \tilde{w}}{\partial \bar{r}}+\tilde{w} \frac{\partial \tilde{w}}{\partial \bar{z}}\right)= & -\frac{\partial \bar{p}}{\partial \bar{z}}+\frac{1}{\overline{\bar{r}}} \frac{\partial}{\partial \bar{r}}\left(\bar{r} S_{\bar{r} \bar{z}}\right)+\frac{\partial}{\partial \bar{z}}\left(\bar{r} S_{\bar{z} \bar{z})}\right. \\
& +\rho g \widehat{\alpha}\left(\tilde{T}-\tilde{T}_{0}\right)+\rho g \widehat{\alpha}_{c}\left(\tilde{C}-\tilde{C}_{0}\right) \\
\left(\tilde{u} \frac{\partial \tilde{T}}{\partial \bar{r}}+\tilde{w} \frac{\partial \tilde{T}}{\partial \bar{z}}\right)= & \alpha\left(\frac{\partial^{2} \tilde{T}}{\partial \bar{r}^{2}}+\frac{1}{\bar{r}} \frac{\partial \tilde{T}}{\partial \bar{r}}+\frac{\partial^{2} \tilde{T}}{\partial \bar{z}^{2}}\right) \\
& +\tau\left[D_{B}\left(\frac{\partial \tilde{C}}{\partial \bar{r}} \frac{\partial \tilde{T}}{\partial \bar{r}}+\frac{\partial \tilde{C}}{\partial \bar{z}} \frac{\partial \tilde{T}}{\partial \bar{z}}\right)\right. \\
& \left.+\frac{D_{\tilde{T}}}{\tilde{T}_{0}}\left\{\left(\frac{\partial \tilde{T}}{\partial \bar{r}}\right)^{2}+\left(\frac{\partial \tilde{T}}{\partial \bar{z}}\right)^{2}\right\}\right] \\
& +\frac{D_{\tilde{T}}}{\tilde{T}_{0}}\left(\frac{\partial^{2} \tilde{T}}{\partial \bar{r}^{2}}+\frac{1}{\bar{r}} \frac{\partial \tilde{T}}{\partial \bar{r}}+\frac{\partial^{2} \tilde{T}}{\partial \bar{z}^{2}}\right) \\
\left(\tilde{u} \frac{\partial \tilde{C}}{\partial \bar{r}}+\tilde{w} \frac{\partial \tilde{C}}{\partial \bar{z}}\right)= & D_{B}\left(\frac{\partial^{2} \tilde{C}}{\partial \bar{r}^{2}}+\frac{1}{\bar{r}} \frac{\partial \tilde{C}}{\partial \bar{r}}+\frac{\partial^{2} \tilde{C}}{\partial \bar{z}^{2}}\right)
\end{aligned}
$$

In the presented equations, $\tau=\frac{(\rho c)_{p}}{(\rho c)_{f}}$ describes the ratio between the effective heat capacity of the nanoparticle material and heat capacity of the fluid, $D_{\widehat{B}}$ as the Brownian diffusion coefficient, $D_{\widehat{T}}$ as the thermophoretic diffusion coefficient, $\widehat{\alpha}_{t}$ as the coefficient of thermal expansion, and $\widehat{\alpha}_{c}$ as the coefficient of thermal expansion with nanoconcentration.

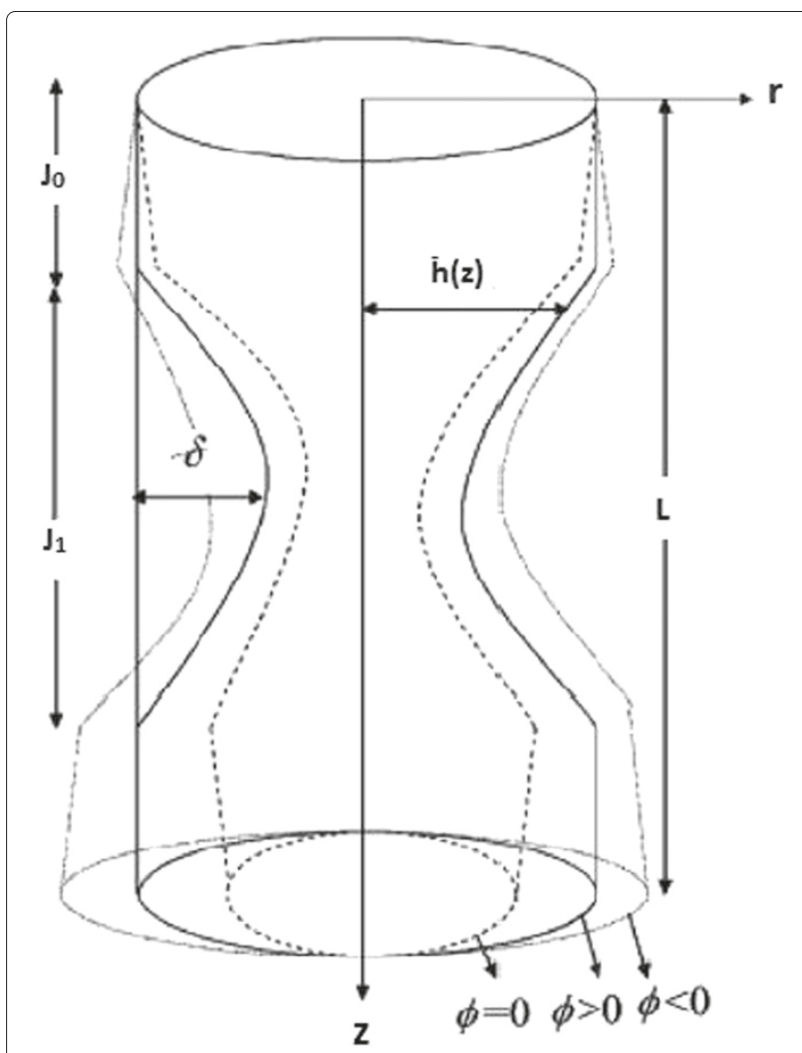

Figure 1 Geometry of a nonsymmetric stenosis in the artery.

The geometry of stenosis is defined as follows [8]:

$$
\begin{aligned}
\overline{\hat{h}}(z) & =Q(z)\left[1-\eta\left(J_{1}^{n-1}\left(\bar{z}-J_{0}\right)-\left(\bar{z}-J_{0}\right)^{n}\right)\right] \\
J_{0} & \leq \bar{z} \leq J_{0}+J_{1} \\
& =Q(z), \text { otherwise }
\end{aligned}
$$

with

$$
Q(z)=Q_{0}+\zeta \bar{z},
$$

where $Q(z)$ be the radius of the tapered arterial section, $Q_{0}$ be the radius of the non-tapered arterial section, $\zeta$ be the tapering parameter, $J_{1}$ be the stenosis length, and $J_{0}$ indicates its place. The parameter $\eta$ is defined as follows:

$$
\eta=\frac{\delta^{*} n^{n-1}}{Q_{0} J_{1}^{n}(n-1)}
$$

where $\delta$ denotes the maximum height of the stenosis located as follows:

$$
\bar{z}=J_{0}+\frac{J_{1}}{n^{\frac{1}{n-1}}},
$$




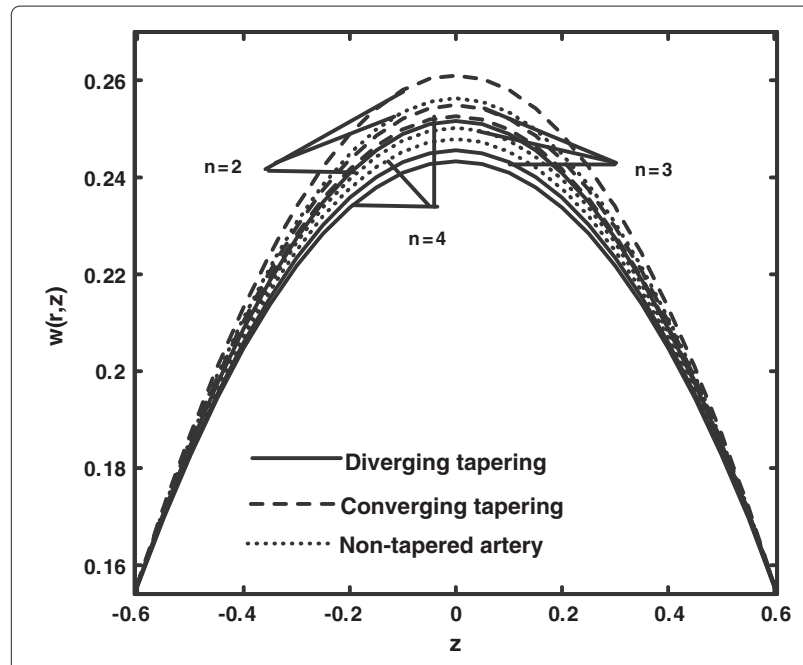

Figure 2 Variation of velocity profile for $F=0.06, J_{3}=0.03$, $n=2, \alpha=0.6, \beta=0.4, N_{t}=0.9, N_{b}=0.9, B_{r}=2, z=0.07$, $G_{r}=2$.

Non-dimensional variables are as follows:

$$
\begin{aligned}
r & =\frac{\bar{r}}{Q_{o}}, z=\frac{\bar{z}}{J_{1}}, w=\frac{\tilde{w}}{u_{0}}, u=\frac{J_{1} \tilde{u}}{u_{0} \delta}, p=\frac{Q_{0} \bar{p}}{u_{0} J_{1} \mu}, \hat{h}=\frac{\hat{h}}{Q_{0}}, \\
R_{e} & =\frac{J_{1} u_{0} \rho}{\mu}, S_{r r}=\frac{J_{1} \bar{S}_{r r}}{u_{0} \mu}, S_{r z}=\frac{Q_{0} \bar{S}_{r z}}{u_{0} \mu}, S_{z z}=\frac{J_{1} \bar{S}_{z z}}{u_{0} \mu}, \\
S_{\theta \theta} & =\frac{J_{1} \bar{S}_{\theta \theta}}{u_{0} \mu}, \\
\lambda_{2} & =\frac{\lambda_{2}^{\star} u_{0}}{J_{1}}, \theta=\frac{\tilde{T}-\tilde{T}_{0}}{\tilde{T}_{0}}, \sigma=\frac{\tilde{C}-\tilde{C}_{0}}{\tilde{C}_{0}}, N_{t}=\frac{(\rho c)_{p} D_{\tilde{T}}}{(\rho c)_{f} \alpha},
\end{aligned}
$$

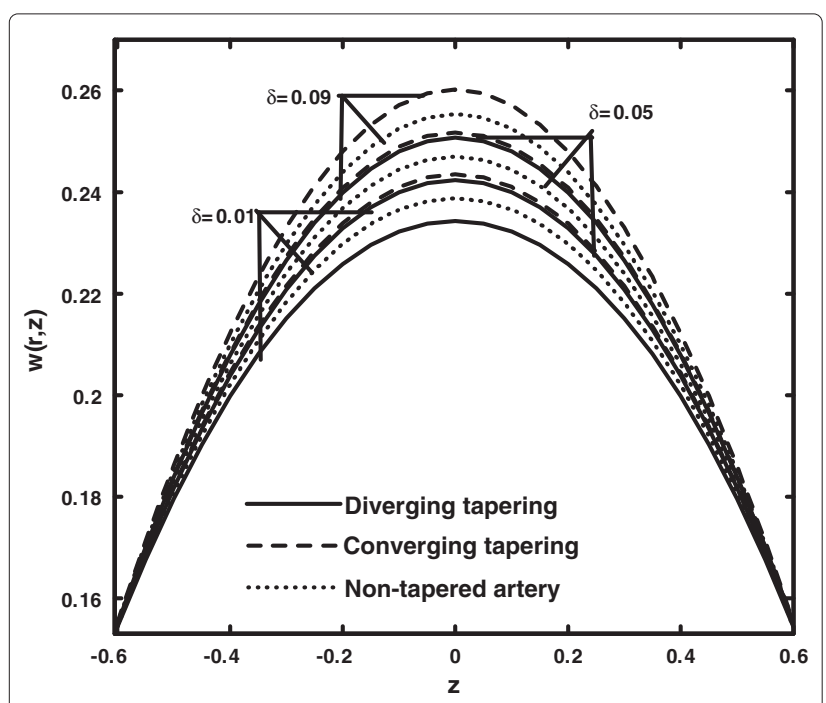

Figure 3 Variation of velocity profile for $F=0.06, J_{3}=0.03$, $\delta=0.09, \alpha=0.6, \beta=0.4, N_{t}=0.9, N_{b}=0.9, B_{r}=2$, $z=0.07, G_{r}=2$.

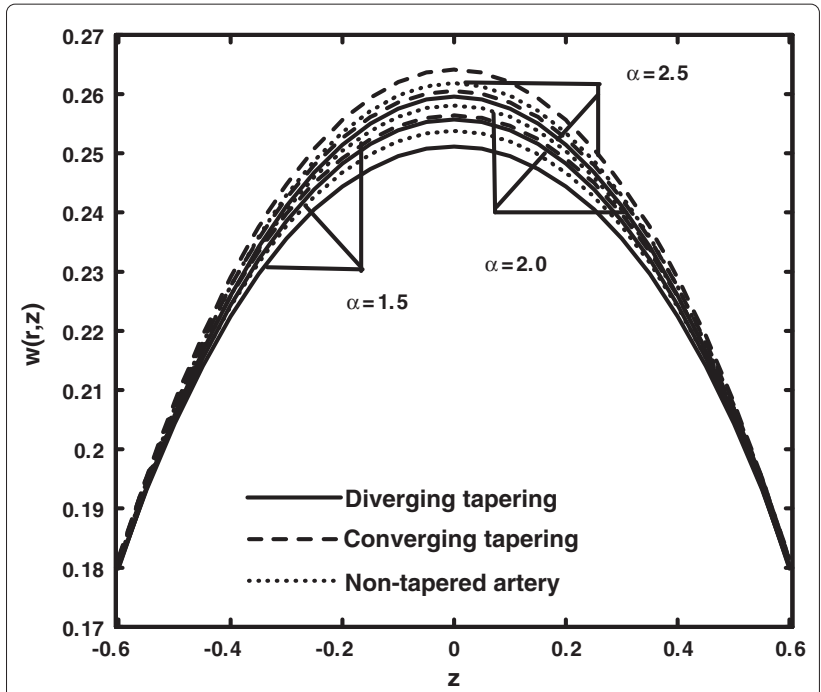

Figure 4 Variation of velocity profile for $F=0.07, J_{3}=0.03$, $n=2, \delta=0.01, \beta=0.4, N_{t}=0.9, N_{b}=0.9, B_{r}=2$, $z=0.07, G_{r}=2$.

$$
\begin{aligned}
\alpha & =\frac{k}{(\rho c)_{f}}, N_{b}=\frac{(\rho c)_{p} D_{B} \tilde{C}_{0}}{(\rho c)_{f} \alpha}, \alpha_{c}=\frac{\rho g \alpha Q_{0} \tilde{C}_{0}}{\mu u_{0}}, \\
\alpha_{t} & =\frac{\rho g \alpha Q_{0} \tilde{T}_{0}}{\mu u_{0}} .
\end{aligned}
$$

Making use of Equation 10 and after taking the condition, we get the following equation:

$$
\frac{\operatorname{Re} \delta^{*} n^{\left(\frac{1}{n-1}\right)}}{J_{1}} \ll 1
$$

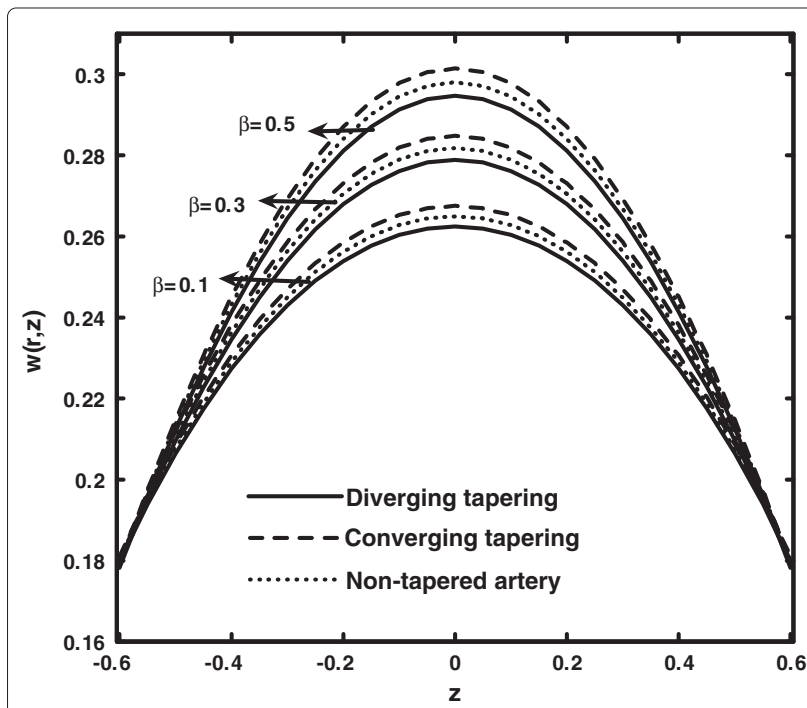

Figure 5 Variation of velocity profile for $F=0.07, J_{3}=0.03$, $n=2, \alpha=0.9, \delta=0.01, N_{t}=0.9, N_{b}=0.9, B_{r}=2$, $z=0.07, G_{r}=2$. 


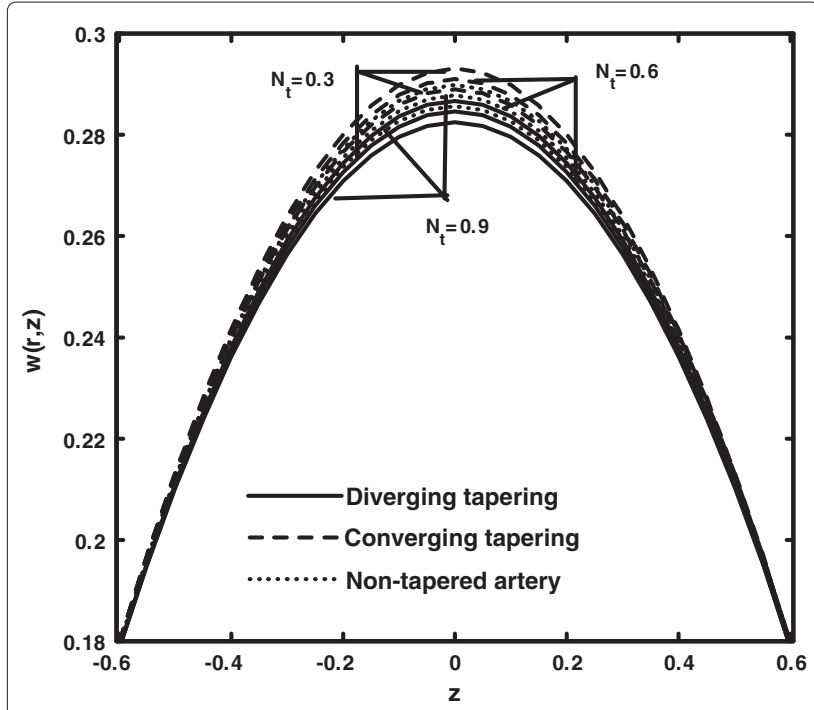

Figure 6 Variation of velocity profile for $F=0.07, J_{3}=0.03$, $n=2, \alpha=0.9, \beta=0.4, \delta=0.01, N_{b}=0.8, B_{r}=1$, $z=0.07, G_{r}=1$.

$$
\frac{Q_{0} n^{\left(\frac{1}{n-1}\right)}}{J_{1}} \backsim O(1) .
$$

Equations 1 to 4 , for the case of mild stenosis $\left(\frac{\delta^{*}}{Q_{o}} \ll 1\right)$, take the the following form:

$$
\begin{aligned}
& \frac{\partial p}{\partial r}=0 \\
& \frac{\partial p}{\partial z}=\frac{1}{r} \frac{\partial}{\partial r}\left[r\left(\alpha\left(\frac{\partial w}{\partial r}\right)+\beta\left(\frac{\partial w}{\partial r}\right)^{3}\right)\right]+G_{r} \theta+B_{r} \sigma
\end{aligned}
$$

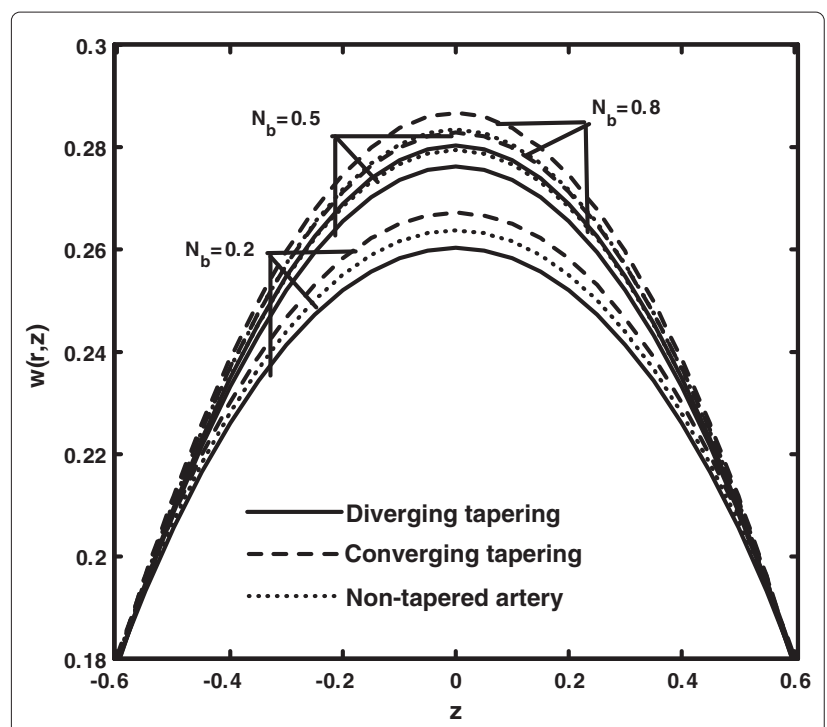

Figure 7 Variation of velocity profile for $F=0.07, J_{3}=0.03$, $n=2, \alpha=0.9, \beta=0.4, N_{t}=0.9, \delta=0.09, B_{r}=1$, $z=0.07, G_{r}=1$.

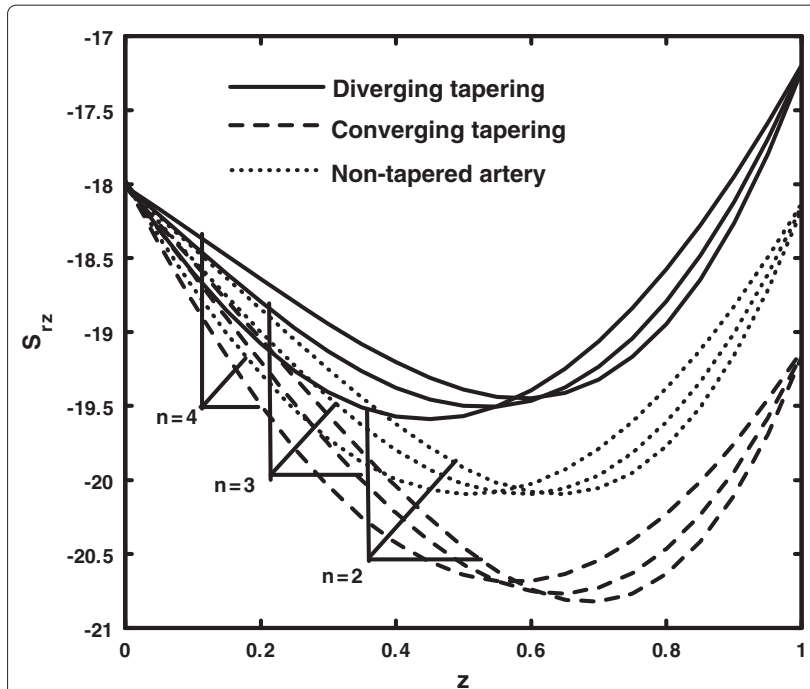

Figure 8 Variation of wall shear stress for $F=0.06, J_{3}=0.01$, $n=2, \alpha=0.9, \beta=0.9, N_{t}=0.9, N_{b}=0.9, B_{r}=1.0$, $G_{r}=1.0$.

$$
\begin{aligned}
& 0=\frac{1}{r} \frac{\partial}{\partial r}\left(r \frac{\partial \theta}{\partial r}\right)+N_{b} \frac{\partial \theta}{\partial r} \frac{\partial \sigma}{\partial r}+N_{t}\left(\frac{\partial \theta}{\partial r}\right)^{2}, \\
& 0=\frac{1}{r} \frac{\partial}{\partial r}\left(\left(r \frac{\partial \sigma}{\partial r}\right)\right)+\frac{N_{t}}{N_{b}}\left(\frac{1}{r} \frac{\partial}{\partial r}\left(r \frac{\partial \theta}{\partial r}\right)\right) .
\end{aligned}
$$

In the above equations, $N_{t}, N_{b}, B_{r}$, and $G_{r}$ are the defined thermophoresis parameter as Brownian motion parameter, as local nanoparticle Grashof number, and

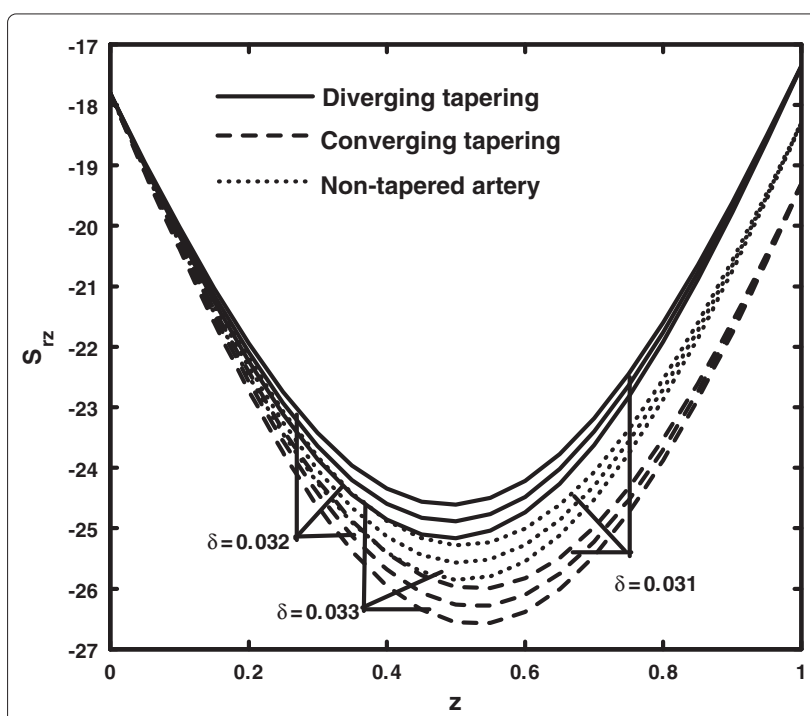

Figure 9 Variation of wall shear stress for $F=0.06, J_{3}=0.01$, $\alpha=0.9, \beta=0.9, B_{r}=1.0, G_{r}=1.0, \delta=0.01, N_{t}=0.9$, $N_{b}=0.9$. 


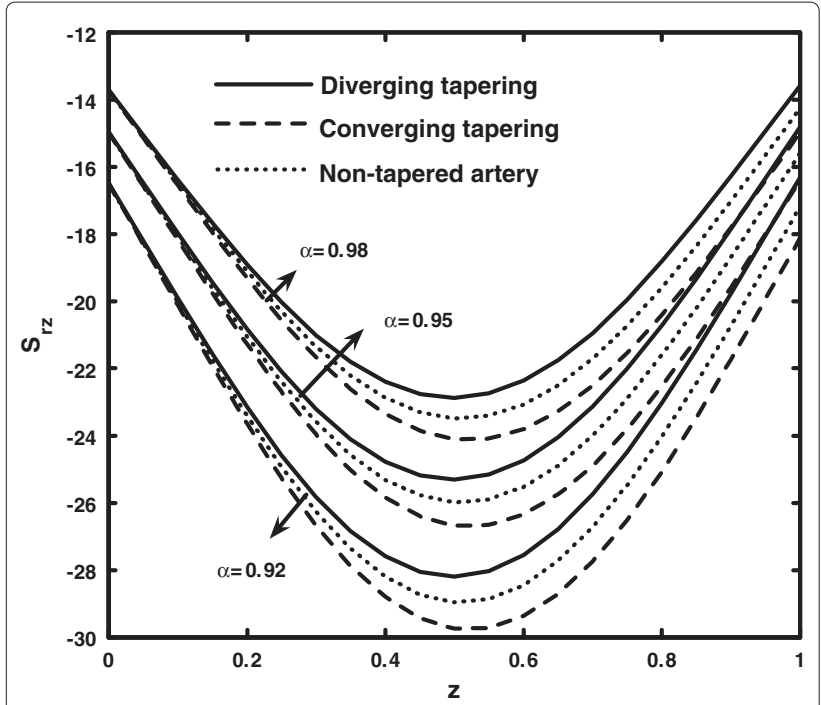

Figure 10 Variation of wall shear stress for $F=0.06, \delta=0.01$, $N_{t}=0.9, B_{r}=1.0, G_{r}=1.0, N_{b}=0.9, J_{3}=0.01, \beta=0.9$.

as local temperature Grashof number, respectively. The boundary conditions are as follows:

$$
\begin{aligned}
\frac{\partial w}{\partial r} & =0, \frac{\partial \theta}{\partial r}=0, \frac{\partial \sigma}{\partial r}=0 \text { at } r=0, \\
w & =0, \theta=0, \sigma=0 \text { at } r=\hat{h},
\end{aligned}
$$

where

$$
\begin{aligned}
\hat{h}(z) & =(1+\zeta z)\left[1-\eta_{1}\left(J_{1}^{n-1}\left(z-J_{0}\right)-\left(z-J_{0}\right)^{n}\right]\right. \\
J_{3} & \leq z \leq J_{3}+1,
\end{aligned}
$$

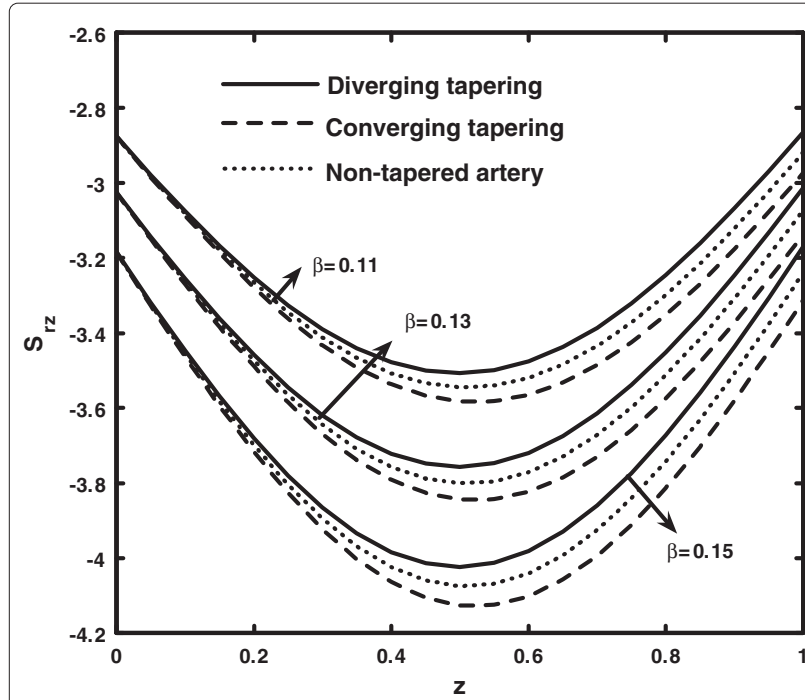

Figure 11 Variation of wall shear stress for $F=0.06, J_{3}=0.01$, $n=2, \alpha=0.9, G_{r}=1.0, N_{t}=0.9, B_{r}=0.9, N_{b}=0.9$.

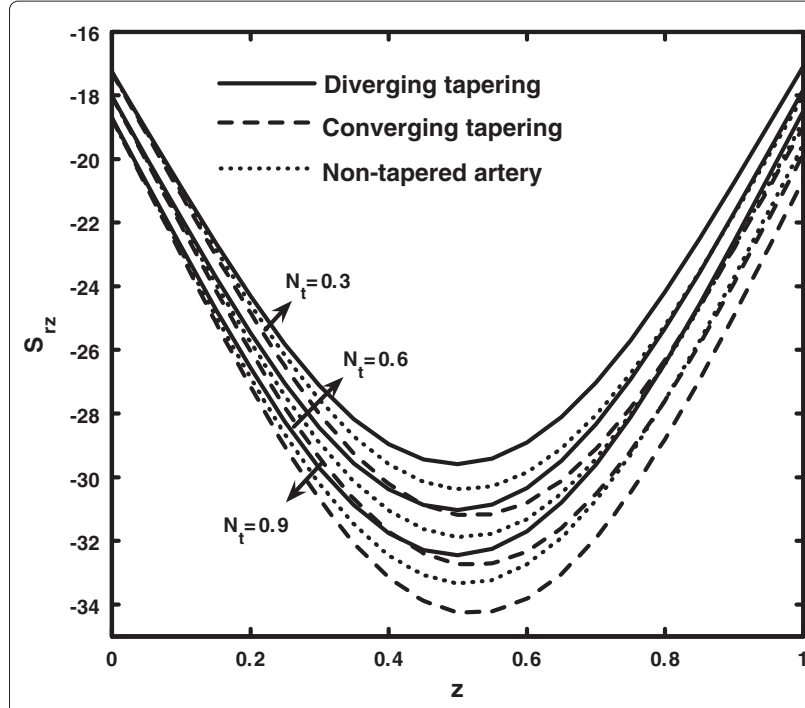

Figure 12 Variation of wall shear stress for $F=0.06, J_{3}=0.01$, $n=2, \alpha=0.9, \beta=0.9, N_{t}=0.9, B_{r}=1.0, G_{r}=1.0$, $\delta=0.01$

and

$$
\eta_{1}=\frac{\delta n^{n-1}}{(n-1)}, \delta=\frac{\delta^{*}}{Q_{o}}, J_{3}=\frac{J_{0}}{J_{1}}, \zeta=\frac{\dot{\zeta} J_{1}}{Q_{o}}, \zeta=\tan \phi,
$$

where $\phi$ is the tapered angle and defined for the nontapered artery $(\phi=0)$, for converging tapering $(\phi<0)$, and for diverging tapering $(\phi>0)$, as described in Figure 1.

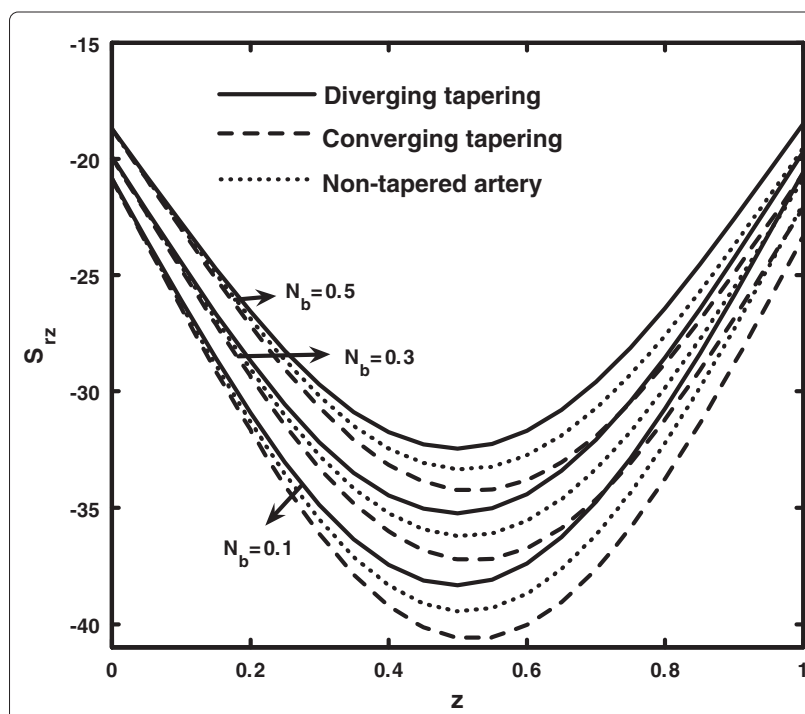

Figure 13 Variation of wall shear stress for $F=0.06, J_{3}=0.01$, $N_{t}=0.9, \beta=0.9, \alpha=0.9, N_{b}=0.9, B_{r}=1.0, G_{r}=1.0$, $\delta=0.01$. 


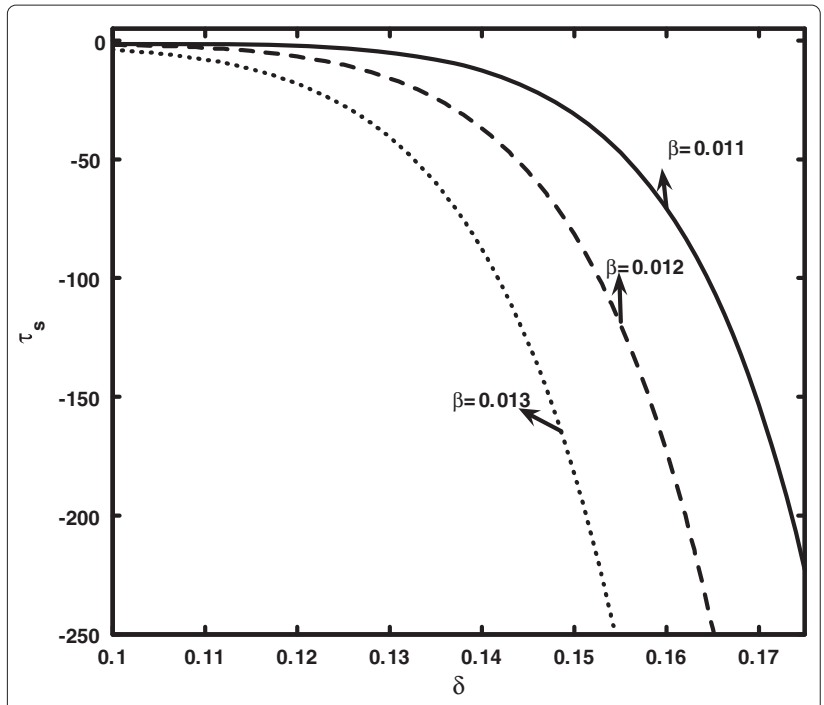

Figure 14 Variation of shear stress at the stenosis throat for $F=0.01, N_{t}=0.09, N_{b}=0.1, B_{r}=0.03, G_{r}=0.05$, $\alpha=0.054$.

\section{Solution of the problem}

\section{Homotopy perturbation solution}

The homotopy perturbation method suggests that we may write Equations 13, 14, and 15 as follows [21,22]:

$$
\begin{aligned}
& \hat{h}(K, \theta)=(1-K)\left[\succeq(\theta)-\succeq\left(\theta_{10}\right)\right] \\
& +\underset{K}{\mathrm{~K}}\left[\mathrm{\iota}(\theta)+N_{b} \frac{\partial \theta}{\partial r} \frac{\partial \sigma}{\partial r}+N_{t}\left(\frac{\partial \theta}{\partial r}\right)^{2}\right] \text {, }
\end{aligned}
$$

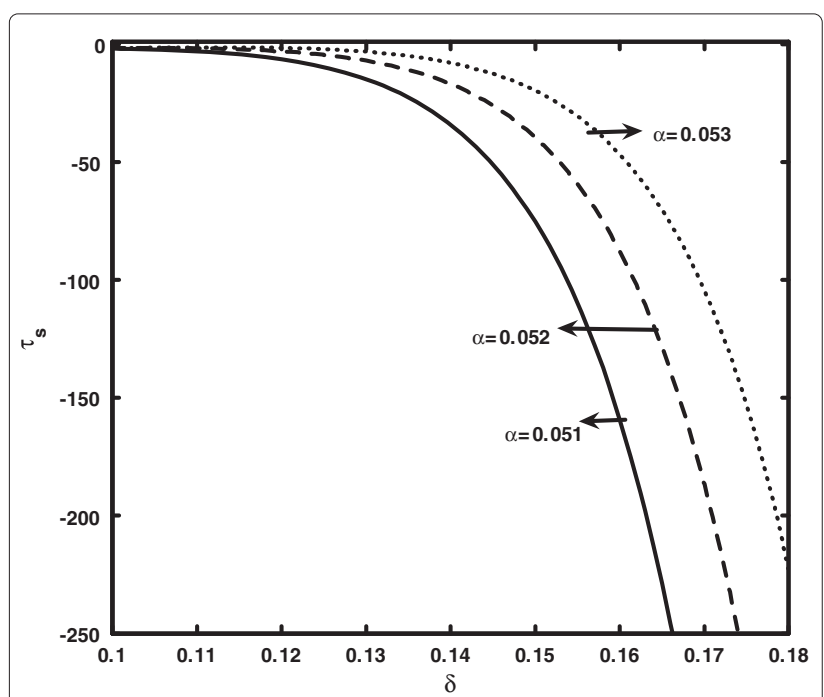

Figure 15 Variation of shear stress at the stenosis throat for $F=0.01, \beta=0.01, N_{t}=0.09, N_{b}=0.1, B_{r}=0.03$, $G_{r}=0.05$.

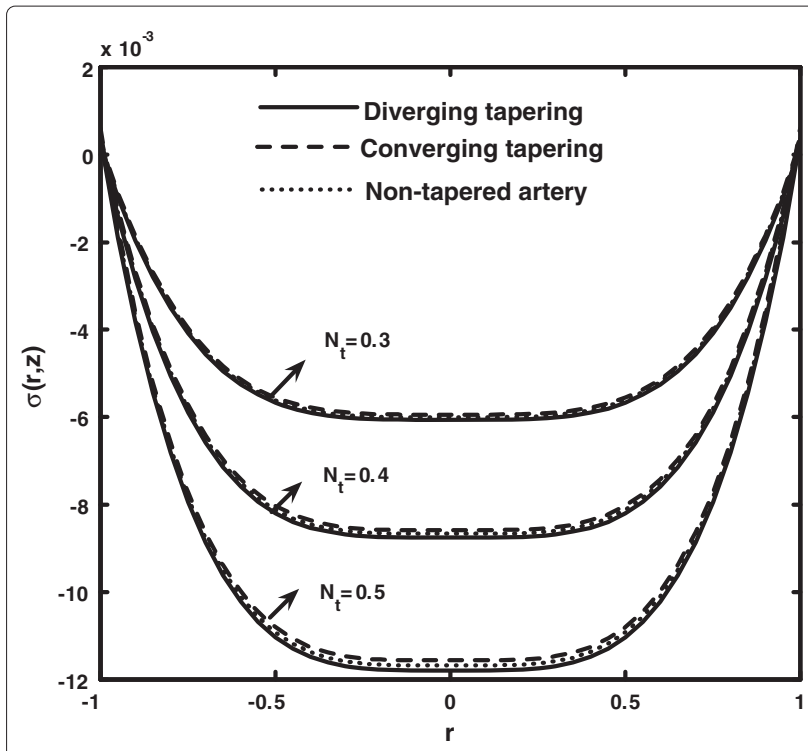

Figure 16 Variation of concentration profile for $\delta=0.01$, $J_{3}=0.0, n=2, z=0.5, N_{b}=0.9$.

$$
\begin{aligned}
& H\left(K_{\xi}, \sigma\right)=(1-\mathrm{K})\left[€(\sigma)-\succeq\left(\sigma_{10}\right)\right] \\
& \left.+\underset{\xi}{K}\left[€(\sigma)+\frac{N_{t}}{N_{b}}\left(\frac{1}{r} \frac{\partial}{\partial r}\left(r \frac{\partial \theta}{\partial r}\right)\right)\right)\right] \text {, } \\
& H(\mathrm{~K}, w)=(1-\mathrm{K})\left[£(w)-Ł\left(w_{10}\right)\right] \\
& +\underset{\xi}{K}\left[\mathrm{~L}(w)+\frac{1}{r} \frac{\partial}{\partial r}\left(r\left(\beta\left(\frac{\partial w}{\partial r}\right)^{3}\right)\right)\right. \\
& \left.+G_{r} \theta+B_{r} \sigma-\frac{\partial p}{\partial z}\right],
\end{aligned}
$$

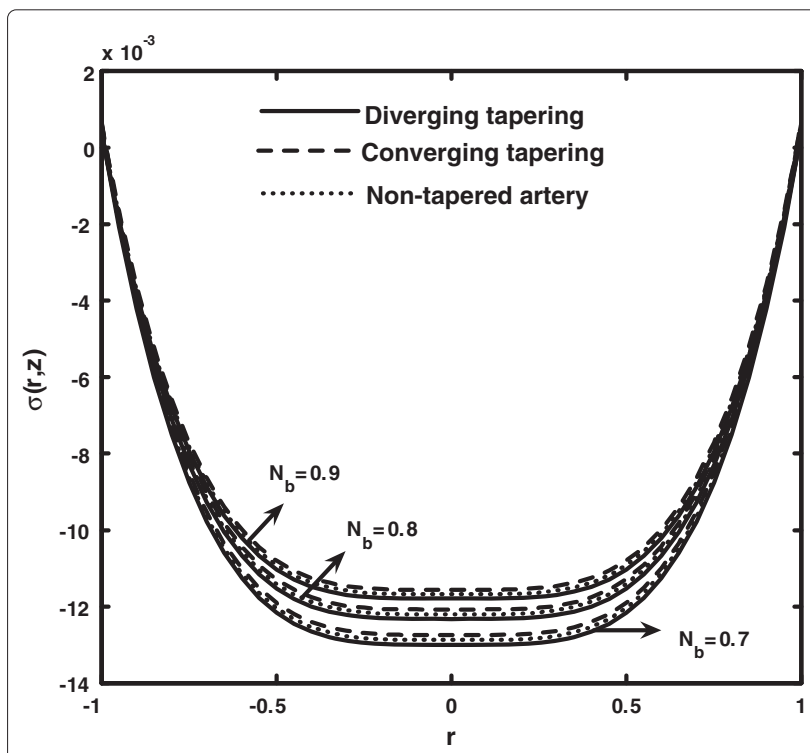

Figure 17 Variation of concentration profile for $\delta=0.5$, $J_{3}=0.0, n=2, z=0.5, N_{t}=0.5$. 


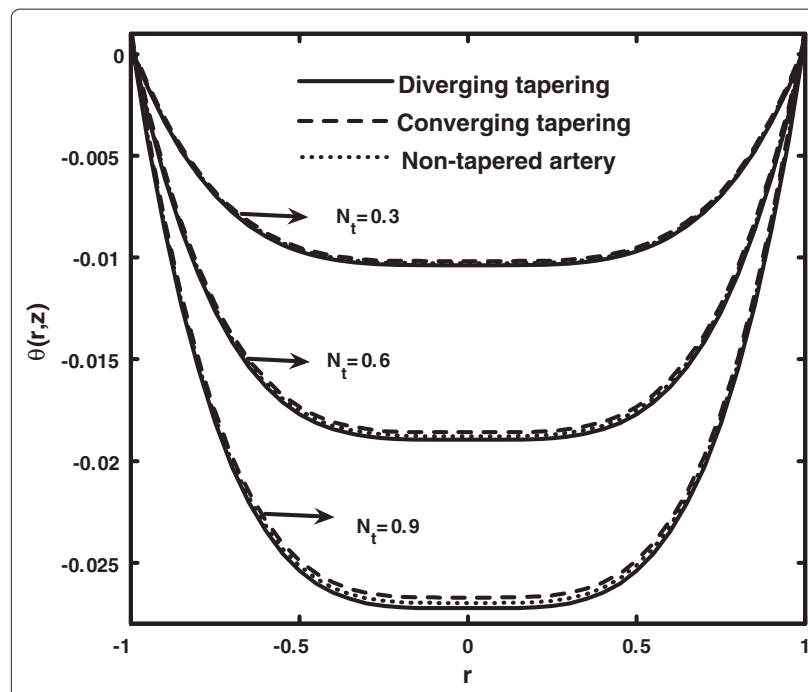

Figure 18 Variation of temperature profile for $\delta=0.5, J_{3}=0.0$, $n=2, z=0.5, N_{b}=0.9$.

taking the following initial guesses:

$$
\begin{aligned}
& \theta_{10}(r, z)=-\left(\frac{r^{2}-\hat{h}^{2}}{4}\right), \sigma_{10}(r, z)=-\left(\frac{r^{2}-\hat{h}^{2}}{4}\right), \\
& w_{10}(r, z)=\frac{1}{\alpha}\left(\frac{r^{2}-\hat{h}^{2}}{4}\right) \frac{d p_{0}}{d z} .
\end{aligned}
$$

We define

$$
\begin{aligned}
& w=w_{0}+K_{\zeta} w_{1}+\aleph_{3}^{2} w_{2}+O\left(K_{s}\right)^{3}, \\
& F=F_{0}+K_{\xi} F_{1}+\aleph^{2} F_{2}+O\left(K^{3}\right)^{3}, \\
& \theta=\theta_{0}+\mathbb{K}_{3} \theta_{1}+\mathbb{K}^{2} \theta_{2}+O\left(K_{s}\right)^{3}, \\
& \sigma=\sigma_{0}+K_{\zeta} \sigma_{1}+\aleph_{3}^{2} \sigma_{2}+O\left(\aleph_{S}\right)^{3} \text {. }
\end{aligned}
$$

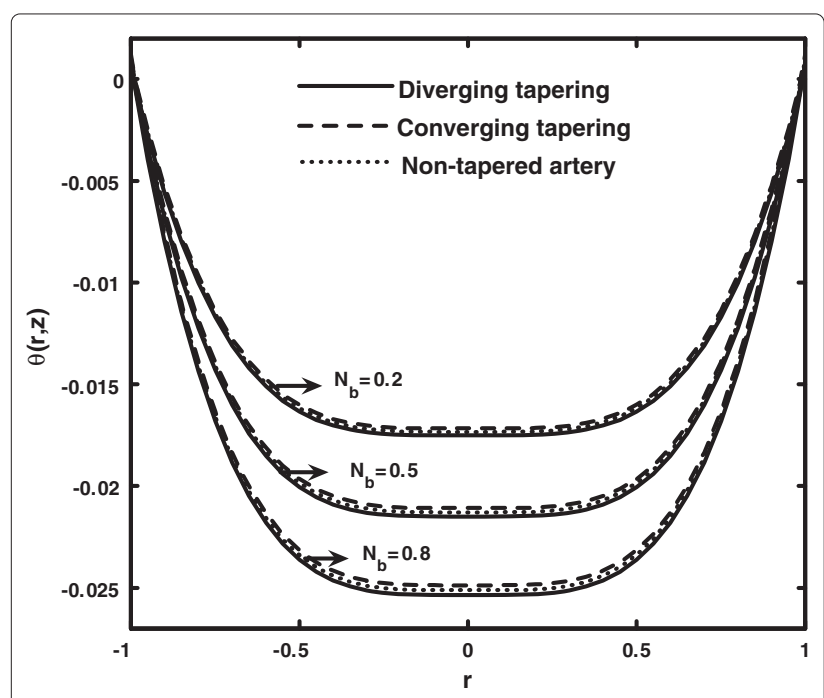

Figure 19 Variation of temperature profile for $\delta=0.5, J_{3}=0.0$, $n=2, z=0.5, N_{t}=0.5$.

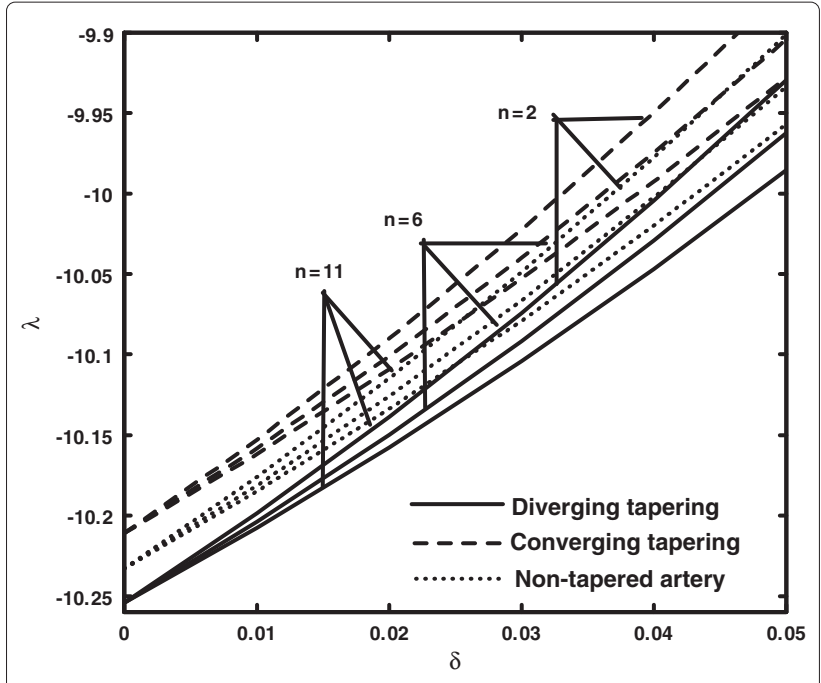

Figure 20 Variation of resistance for $F=0.01, J_{3}=0.09$, $B_{r}=0.3, G_{r}=0.1, L=2, N_{t}=0.01, N_{b}=0.1, \alpha=0.1$, $\beta=0.03$.

Putting Equations 23 to 26 into Equations 19 to 21 and taking $\mathrm{K} \rightarrow 1$, the following form for temperature profile, concentration profile, velocity profile, and pressure gradient are written as follows:

$$
\begin{aligned}
w(r, z)= & \frac{1}{\alpha} \frac{d p}{d z}\left(\frac{r^{2}-\hat{h}^{2}}{4}\right)+\left(\frac{\hat{e}_{20}}{\alpha}+\hat{e}_{10}\right)\left(r^{2}-\hat{h}^{2}\right) \\
& +\left(\frac{\beta \hat{e}_{17}+\hat{e}_{19}}{\alpha}+\hat{e}_{11}\right)\left(r^{4}-\hat{h}^{4}\right) \\
& +\frac{\left(\beta \hat{e}_{16}+\hat{e}_{18}\right)}{\alpha}\left(r^{6}-\hat{h}^{6}\right),
\end{aligned}
$$

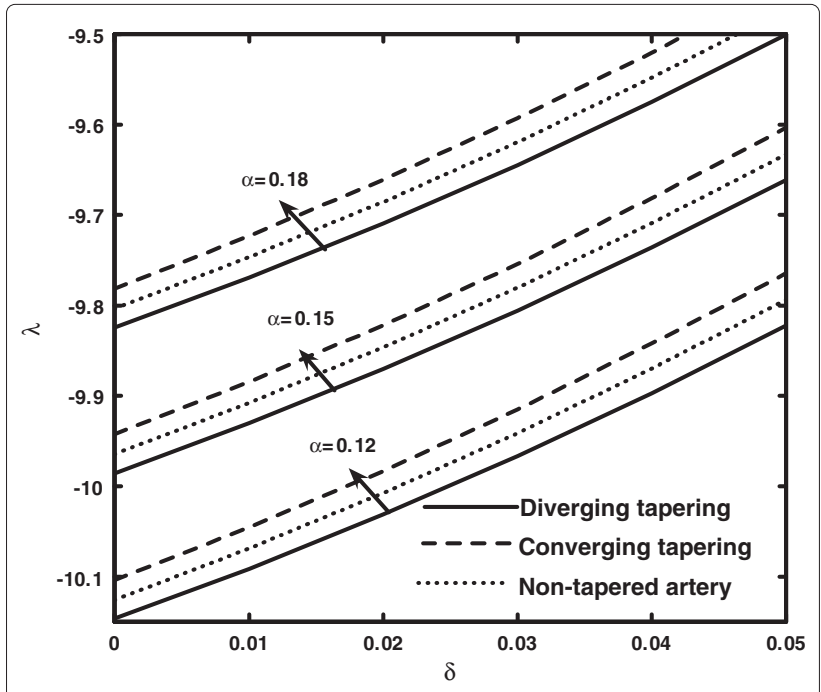

Figure 21 Variation of resistance for $F=0.01, J_{3}=0.09$, $B_{r}=0.3, G_{r}=0.1, n=2, L=2, N_{t}=0.01, N_{b}=0.1$, $\beta=0.01$. 


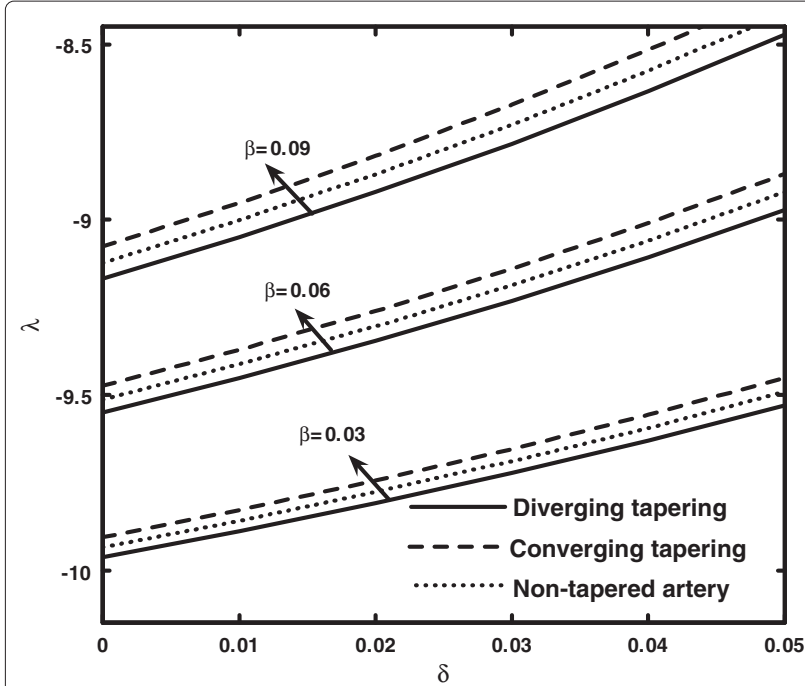

Figure 22 Variation of resistance for $F=0.01, J_{3}=0.09$, $B_{r}=0.3, G_{r}=0.1, n=2, L=2, N_{b}=0.1, \alpha=0.1$, $N_{t}=0.01$.

$$
\begin{aligned}
\theta(r, z)= & -\left(\frac{r^{2}-\hat{h}^{2}}{4}\right)+\left(2 N_{t}+N_{b}\right)\left(\frac{r^{4}-\hat{h}^{4}}{64}\right) \\
& +\left(\frac{r^{2}-\hat{h}^{2}}{4}\right)-\left(\frac{r^{6}-\hat{h}^{6}}{1152}\right) \\
& \left(N_{t}+N_{b}\right)\left(2 N_{t}+N_{b}\right), \\
\sigma(r, z)= & -\left(\frac{r^{2}-\hat{h}^{2}}{4}\right)+\left(1+\frac{N_{t}}{N_{b}}\right)\left(\frac{r^{2}-\hat{h}^{2}}{4}\right) \\
& -\frac{N_{t}}{N_{b}}\left\{\left(\frac{r^{2}-\hat{h}^{2}}{4}\right)-\left(N_{t}+N_{b}\right)\left(\frac{r^{4}-\hat{h}^{4}}{64}\right)\right\},
\end{aligned}
$$

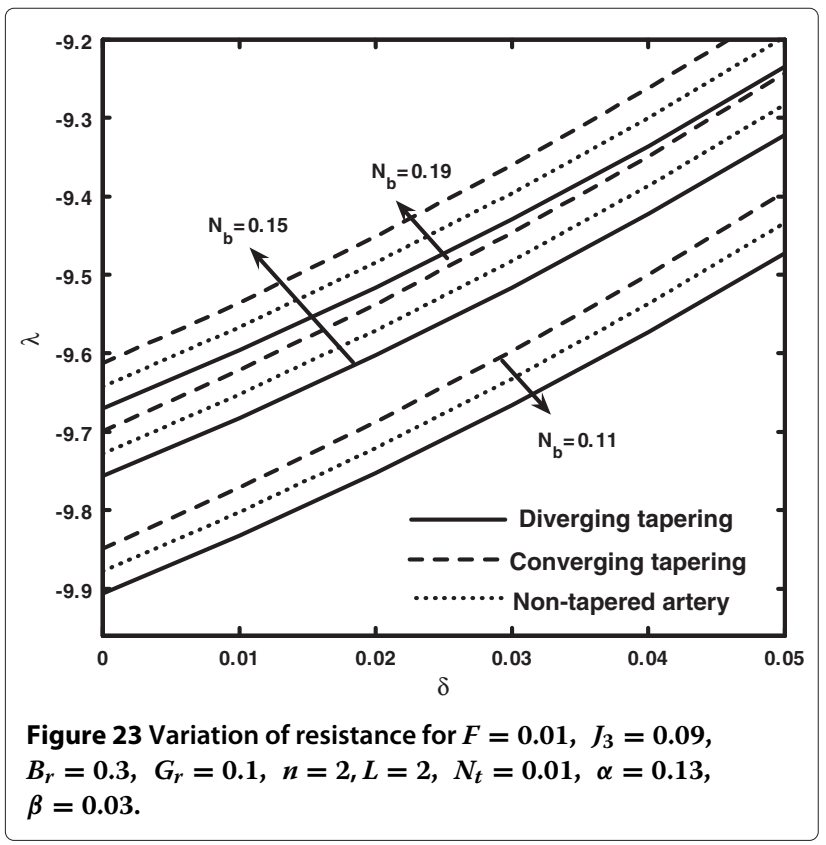

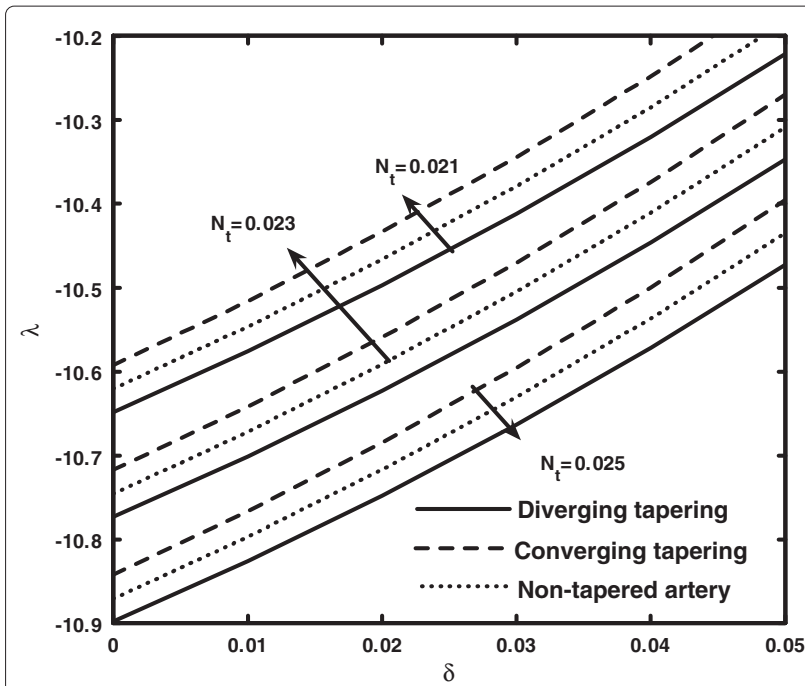

Figure 24 Variation of resistance for $F=0.01, J_{3}=0.09$, $B_{r}=0.3, G_{r}=0.1, n=2, L=2, N_{b}=0.1, \alpha=0.1$, $\beta=0.03$.

$$
\frac{d p}{d z}=-\frac{16 F \alpha}{\hat{h}^{4}}-\frac{16\left(\alpha \hat{e}_{21}+\beta \hat{e}_{22}+\hat{e}_{23}\right)}{\hat{h}^{4}} .
$$

Pressure drop $(\Delta p=p$ at $z=0$ and $\Delta p=-p$ and $z=L)$ through the stenosis between the regions $z=0$ and $z=L$ computed from Equation 30 can be written as follows:

$$
\Delta p=\int_{0}^{L}\left(-\frac{d p}{d z}\right) d z
$$

\section{Resistance impedance}

Using Equation 31, the expression for resistance impedance is given as follows:

$$
\begin{gathered}
\bar{\lambda}=\frac{\Delta p}{F}=\left\{\left.\int_{0}^{J_{0}} E(z)\right|_{\hat{h}=1} d z+\int_{J_{0}}^{J_{0}+J_{1}} E(z) d z\right. \\
\left.+\left.\int_{J_{0}+J_{1}}^{L} E(z)\right|_{\hat{h}=1} d z\right\}
\end{gathered}
$$

where

$$
\begin{aligned}
E(z)= & \frac{16 \alpha}{\hat{h}^{4}}+\frac{16\left(\alpha \hat{e}_{21}+\beta \hat{e}_{22}+\hat{e}_{23}\right)}{\hat{h}^{4} F}, \\
\bar{\lambda}= & \left\{\left(L-J_{1}\right)\left(16 \alpha+\frac{\left.16\left(\alpha \hat{e}_{21}+\beta \hat{e}_{22}+\hat{e}_{23}\right)\right|_{\hat{h}=1}}{F}\right)\right. \\
& \left.+\int_{J_{0}}^{J_{0}+J_{1}} E(z) d z\right\},
\end{aligned}
$$




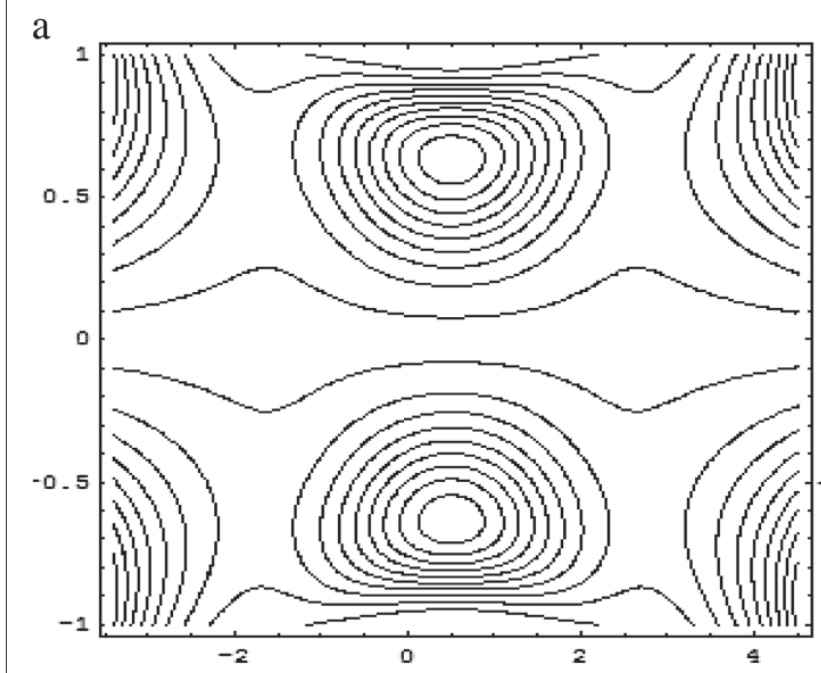

Figure 25 Stream lines for different values of (a) $\boldsymbol{F}=\mathbf{0 . 2 0}$ and (b)

$J_{3}=0.01, \delta=0.01, n=2, N_{t}=0.5, B_{r}=3.5, G_{r}=2.7$.

\section{Expression for the wall shear stress}

Dimensionless shear stress is defined as follows:

$$
\tilde{S}_{r z}=\left(\alpha\left(\frac{\partial w}{\partial r}\right)+\beta\left(\frac{\partial w}{\partial r}\right)^{3}\right) .
$$

or

$$
\tilde{S}_{r z}=\left.\left[\left(\alpha\left(\frac{\partial w}{\partial r}\right)+\beta\left(\frac{\partial w}{\partial r}\right)^{3}\right)\right]\right|_{r=\hat{h}},
$$

$$
\tilde{S}_{r z}=\frac{\hat{h}}{2} \frac{d p}{d z}\left(1+\frac{\beta \hat{h}^{2}}{4 \alpha^{3}}\left(\frac{d p}{d z}\right)^{2}\right)+\hat{e}_{24}
$$

Using Equation 27, we obtain the following:

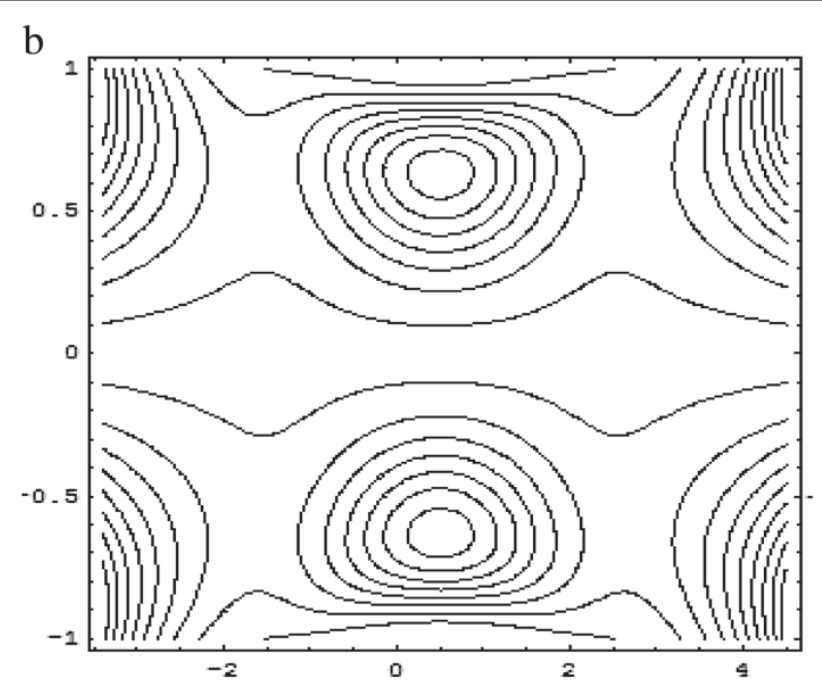

0.21. Other parameters are $N_{b}=0.011, \phi=\pi, \alpha=0.90, \beta=2.4$,

The shearing stress at the stenosis throat located at $z=$ $\frac{J_{0}}{J_{1}}+\frac{1}{n^{\frac{1}{n-1}}}$ is defined as follows:

$$
\tilde{\tau}_{s}=\left.\tilde{S}_{r z}\right|_{\hat{h}=1-\delta}
$$

or

$$
\tilde{\tau}_{s}=\frac{\hat{h}}{2} \frac{d p}{d z}\left(1+\frac{\beta \hat{h}^{2}}{4 \alpha^{3}}\left(\frac{d p}{d z}\right)^{2}\right)+\left.\hat{e}_{24}\right|_{\hat{h}=1-\delta} .
$$

The final expression for $\lambda, S_{r z}$, and $\tau_{s}$ can be defined as follows:

$S_{r z}=\frac{\hat{h}}{8 F} \frac{d p}{d z}\left(1+\frac{\beta \hat{h}^{2}}{4 \alpha^{3}}\left(\frac{d p}{d z}\right)^{2}\right)+\frac{\hat{e}_{24}}{4 F}$,
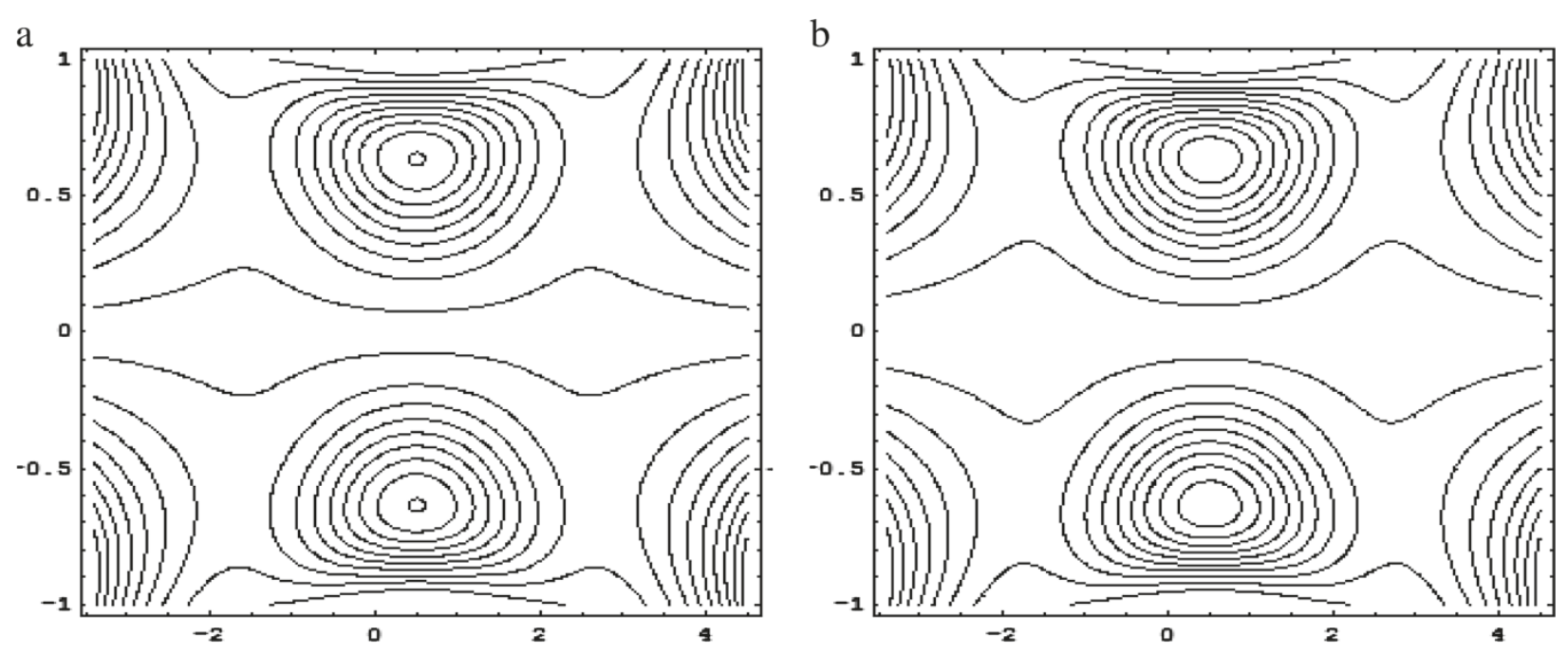

Figure 26 Stream lines for different values of (a) $\beta=2.3$ and (b) $\beta=2.5$. 


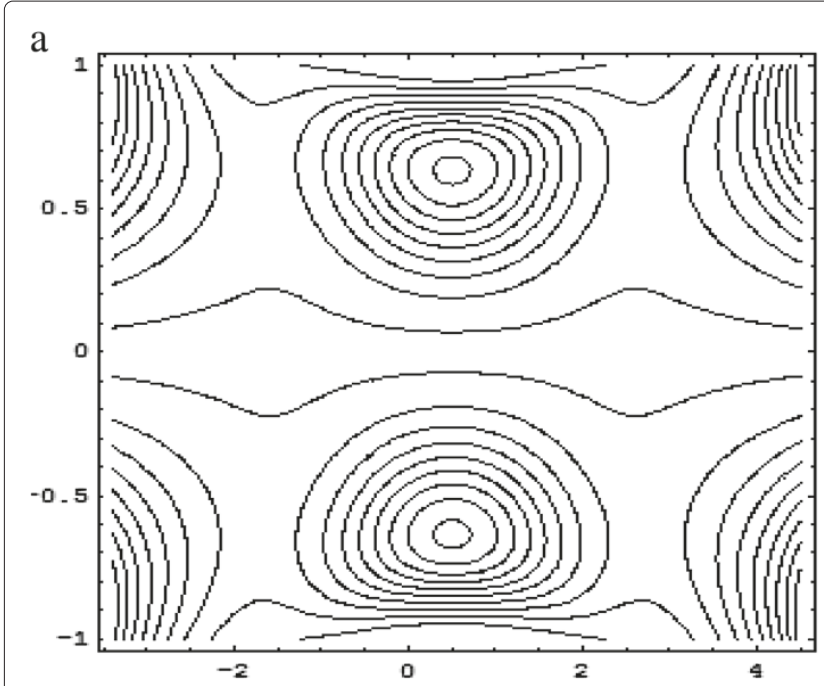

Figure 27 Stream lines for different values of $(\mathbf{a}) \boldsymbol{\alpha}=\mathbf{0 . 9 1}$ and (b) $\boldsymbol{\alpha}=\mathbf{0 . 9 2}$. Other parameters are $N_{b}=0.011, \phi=\pi, F=0.21, \beta=2.4$,

b

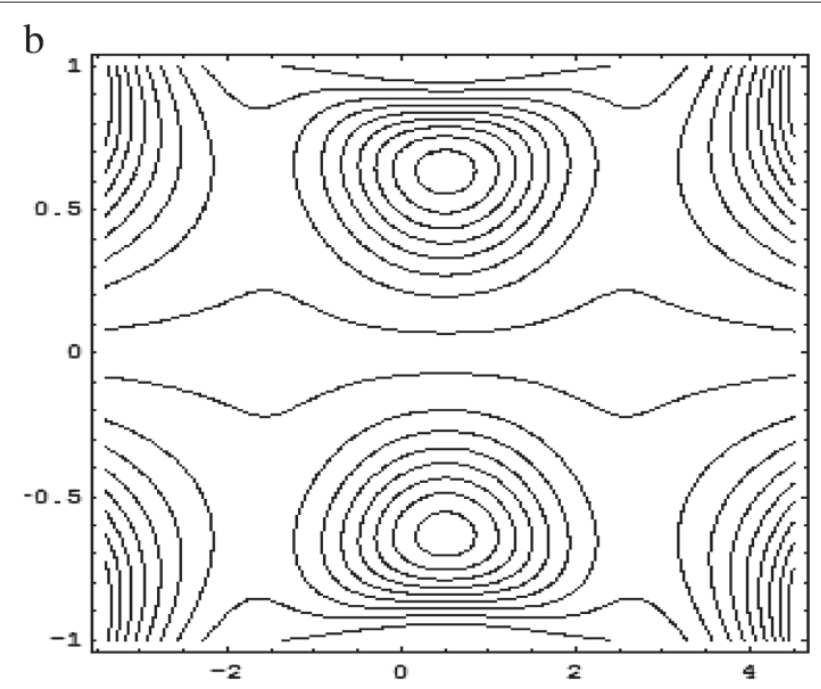

in which

$$
\lambda=\frac{\bar{\lambda}}{\lambda_{0}}, S_{r z}=\frac{\tilde{S}_{r z}}{\tau_{0}}, \tau_{s}=\frac{\tilde{\tau}_{s}}{\tau_{0}}, \lambda_{0}=3 L, \tau_{0}=4 F .
$$

\section{Results and discussion}

The quantitative results of the $\alpha, \beta, n, \delta, N_{t}, a n d N_{b}$ for diverging tapering, converging tapering, and non-tapered arteries are observed physically in Figures 2, 3, 4, 5, 6, 7, $8,9,10,11,12,13,14,15,16,17,18,19,20,21,22,23$, and 24. Variations of velocity profile for $\alpha, \beta, n, \delta, N_{t}$, and $N_{b}$ for the cases of converging tapering, diverging tapering, and non-tapered arteries are displayed in Figures 2, 3, $4,5,6$, and 7. In Figures 2, 3, 4, 5, 6, and 7, it is analyzed
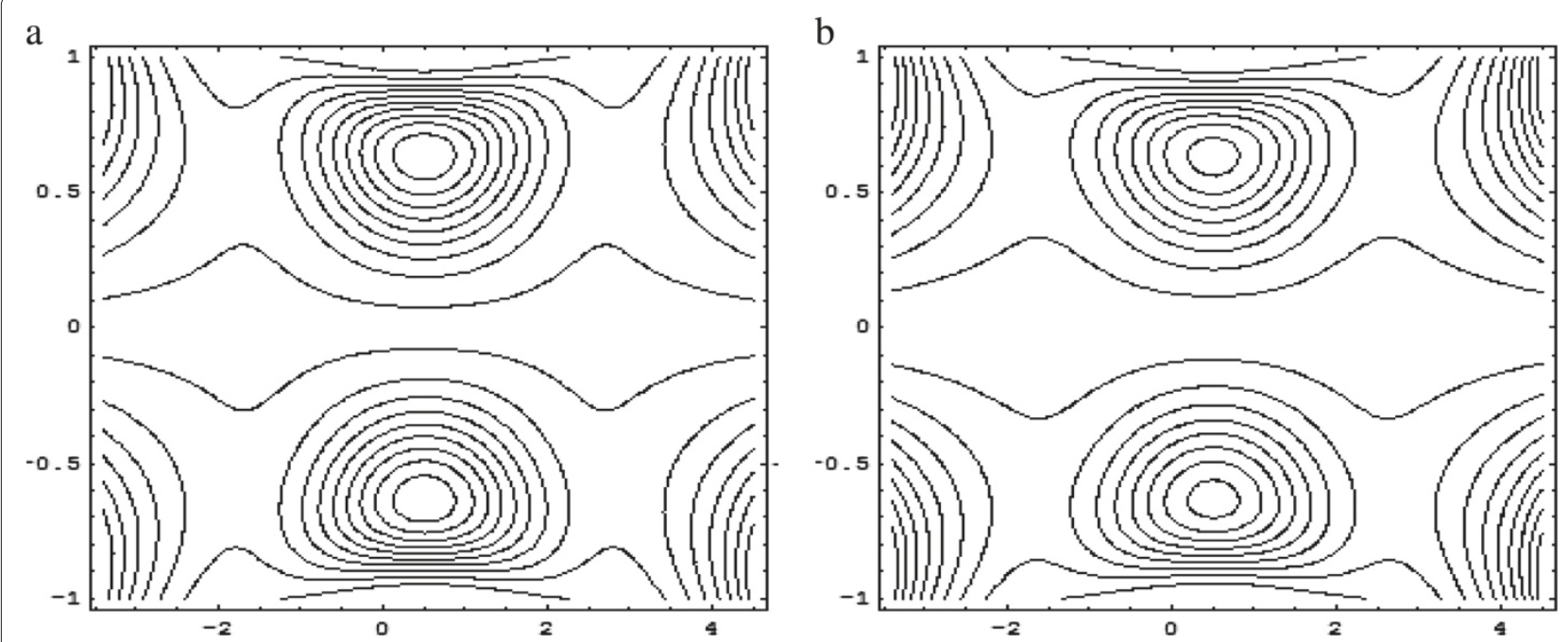

Figure 28 Stream lines for different values of (a) $\boldsymbol{N}_{\boldsymbol{t}}=\mathbf{0 . 4}$ and (b) $\boldsymbol{N}_{\boldsymbol{t}}=\mathbf{0 . 6}$. Other parameters are $N_{b}=0.011, \phi=\pi, F=0.21, \alpha=0.90$, $J_{3}=0.01, \delta=0.01, n=2, \beta=2.4, B_{r}=3.5, G_{r}=2.7$. 


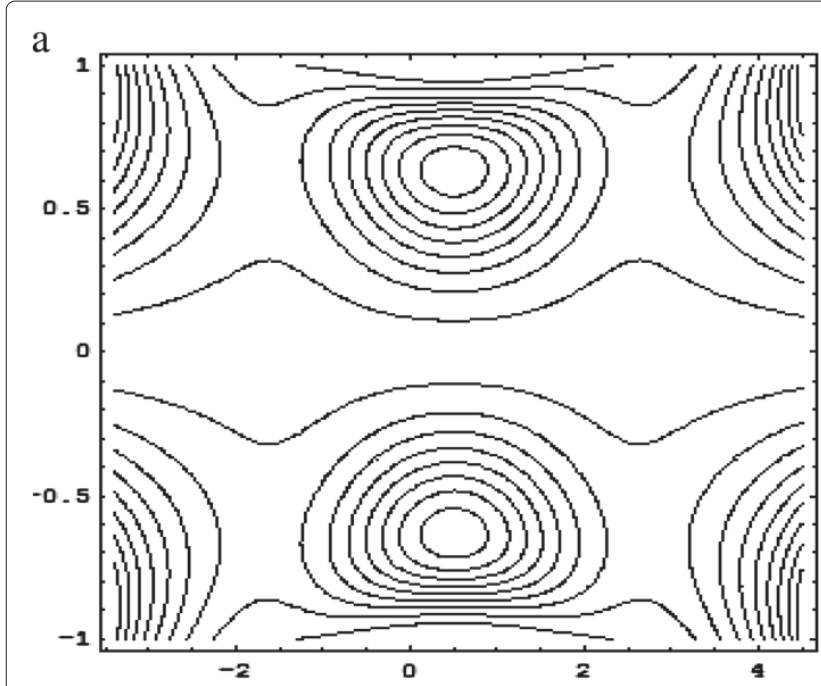

Figure 29 Stream lines for different values of (a) $\boldsymbol{N}_{\boldsymbol{b}}=\mathbf{0 . 0 1 0}$ and (b) $\boldsymbol{N}_{\boldsymbol{b}}=\mathbf{0 . 0 1 1}$. Other parameters are $N_{t}=0.6, \phi=\pi, F=0.21, \alpha=0.90$, $J_{3}=0.01, \delta=0.01, n=2, \beta=2.4, B_{r}=3.5, G_{r}=2.7$.

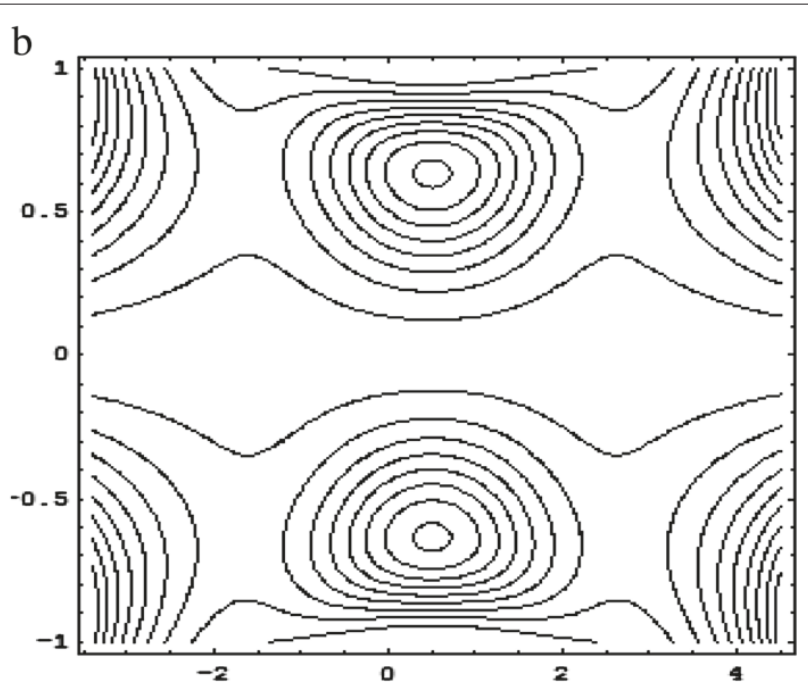

b

while it increases with an increase in Prandtl parameter $\alpha$ and Brownian motion parameter $N_{b}$. Figures 14 and 15 depict variations of the shearing stress at the stenosis throat $\tau_{s}$ with $\delta$. In these figures, it is analyzed that the shearing stress at the stenosis throat decreases with an increase in $\beta$ and increases with an increase in $\alpha$. Figures 16 and 17 show variations of concentration profile for the Brownian motion parameter $N_{b}$ and thermophoresis parameter $N_{t}$. It is observed that with an increase in the Brownian motion parameter $N_{b}$, the concentration profile increases, while it decreases with an increase in the thermophoresis parameter $N_{t}$ and the concentration profile gives a larger value for the converging tapering
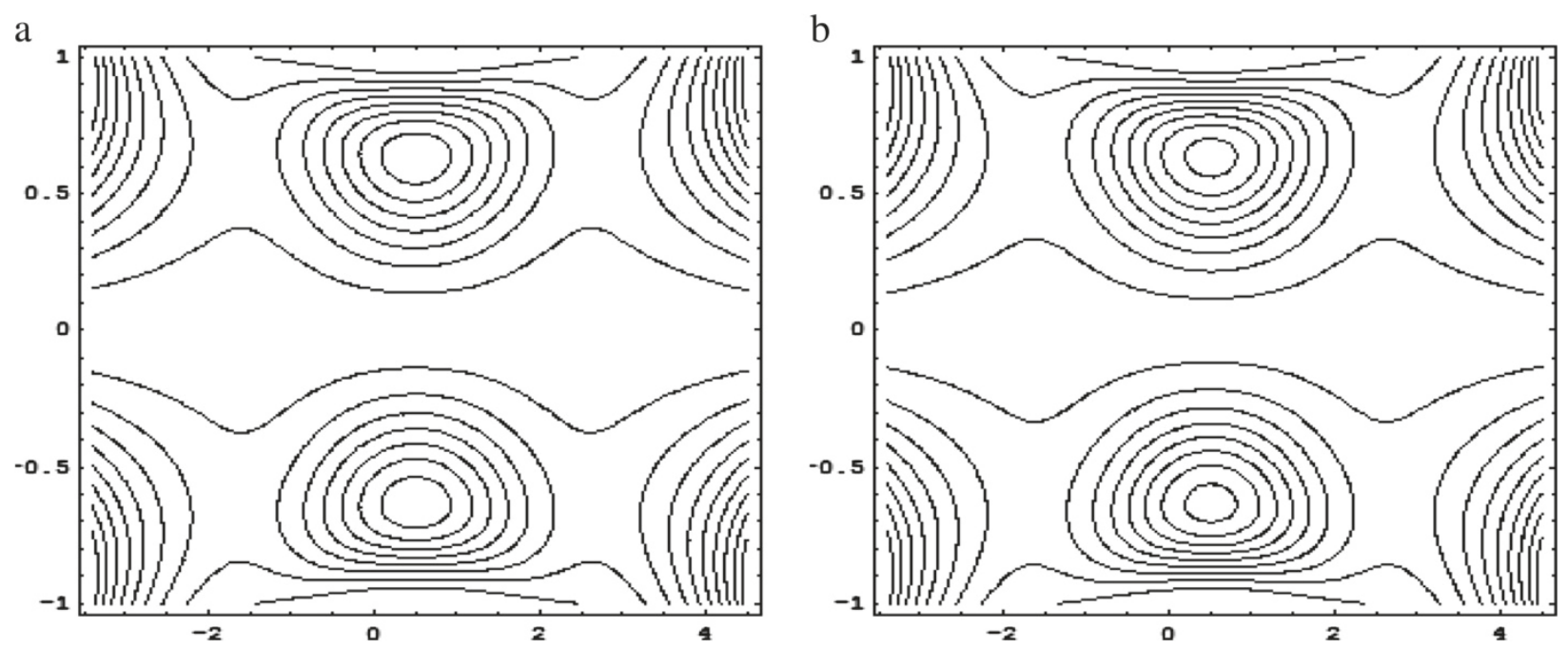

Figure 30 Stream lines for different values of (a) $\boldsymbol{B}_{\boldsymbol{r}}=\mathbf{3 . 4}$ and (b) $\boldsymbol{B}_{\boldsymbol{r}}=\mathbf{3 . 6}$. Other parameters are $N_{t}=0.6, \phi=\pi, F=0.21, \alpha=0.90$, $J_{3}=0.01, \delta=0.01, n=2, \beta=2.4, G_{r}=2.7, N_{b}=0.011$. 
artery. Figures 18 and 19 depict variations of the temperature profile for the Brownian motion parameter $N_{b}$ and thermophoresis parameter $N_{t}$. It is observed that with an increase in the Brownian motion parameter $N_{b}$ and the thermophoresis parameter $N_{t}$, the temperature profile decreases. Figures 20, 21, 22, 23, and 24 describe the impedance resistance increases for non-tapered, diverging tapering, and converging tapering arteries when we increase the Prandtl parameters, $\alpha$, and $\beta$, and Brownian motion parameter $N_{b}$, while it decreases with an increase in thermophoresis parameters, $N_{t}$ and $n$.

\section{Trapping}

Trapping phenomena can be analyzed in Figures 24, 25, $26,27,28,29,30,31$. It is analyzed that with an increase in flow rate $F$, the number of trapping bolus increases. We also observed that with an increase in $\beta$, the size of trapping bolus increases, while the number of trapping bolus increases with an increase in $\alpha$. It is also observed that with an increase in the Brownian motion parameter $N_{b}$, the number of trapping bolus increases, while with an increase in the thermophoresis parameter $N_{t}$ the number of trapping bolus decreases. It is also analyzed that with local nanoparticle Grashof numbers $B_{r}$ and $G_{r}$, the size of trapping bolus decreases.

\section{Conclusions}

The main points of the study that were examined are as follows:

1. It is analyzed that with an increase in the thermophoresis parameter $N_{t}$ and stenosis shape $n$, the velocity profile decreases, while the velocity profile increases with an increase in the maximum height of the stenosis $\delta$, the Brownian motion parameter $N_{b}$, and Prandtl parameters $\alpha$ and $\beta$.

2. It is analyzed that with an increase in stenosis shape $n$, the thermophoresis parameter $N_{t}$, stenosis height $\delta$, and Prandtl parameter $\beta$, the shear stress decreases, while it increases with an increase in the Brownian motion parameter $N_{b}$ and Prandtl parameter $\alpha$.

3. It is analyzed that the shearing stress at the stenosis throat decreases with an increase in $\beta$ and increases with an increase in $\alpha$.

4. It is observed that with an increase in the Brownian motion parameter $N_{b}$, the concentration profile increases, while it decreases with an increase in the thermophoresis parameter $N_{t}$.

5. It is observed that with an increase in the Brownian motion parameter, the temperature profile decreases, while with an increase in the thermophoresis parameter, the temperature profile increases.

6. It is observed that with an increase in the Brownian motion parameter $N_{b}$ and the thermophoresis parameter $N_{t}$, the temperature profile decreases.

7. It is analyzed that with an increase in flow rate $F$, Prandtl parameter $\alpha$, and Brownian motion parameter $N_{b}$, the number of trapping bolus increases, while it decreases with an increase in the thermophoresis parameter $N_{t}$.

8. It is also analyzed that the size of trapping bolus decreases with an increase in the local nanoparticle Grashof numbers $B_{r}$ and $G_{r}$, while the size of trapping bolus increases with an increase in the Prandtl parameter $\beta$.
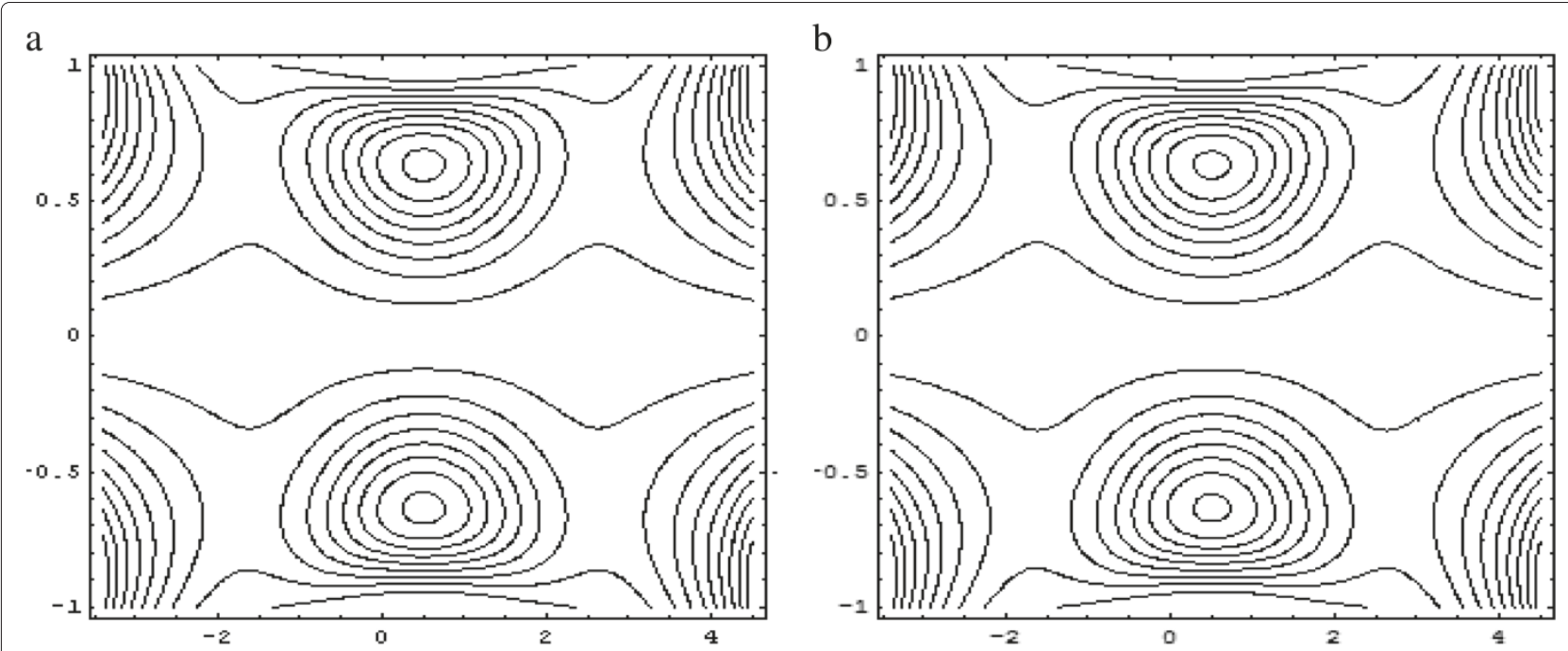

Figure 31 Stream lines for different values of (a) $\boldsymbol{G}_{\boldsymbol{r}}=\mathbf{1 . 5}$ and (b) $\boldsymbol{G}_{\boldsymbol{r}}=\mathbf{2 . 5}$. Other parameters are $N_{t}=0.6, \phi=\pi, F=0.21, \alpha=0.90$, $J_{3}=0.01, \delta=0.01, n=2, \beta=2.4, B_{r}=3.6, N_{b}=0.011$. 


\section{Competing interests}

The authors declare that they have no competing interests.

\section{Authors' contributions}

All authors - SN, SI, and NSA - have contributed in all the sections in the manuscript. All authors read and approved the final manuscript.

\section{Acknowledgments}

The corresponding author is thankful to Quaid-i-Azam University for providing URF to complete this research.

\section{Author details}

'Department of Mathematics, Quaid-i-Azam University 45320, Islamabad 44000 , Pakistan. ${ }^{2}$ DBS\&H, CEME, National University of Sciences and Technology, Islamabad, 44000, Pakistan.

Received: 30 July 2012 Accepted: 29 January 2013

Published: 15 May 2013

\section{References}

1. Thurston, GB: Viscoelasticity of human blood. Biophys. J. 12, 1205-1217 (1972)

2. Chien, S, King, RG, Skalak, R, Usami, S, Copley, AL: Viscoelastic properties of human blood and red cell suspension. Biorheology. 12, 341-346 (1975)

3. Aroesty, J, Gross, JF: Pulsatile flow in small vessels - I Casson theory. Biorheology. 9, 33-42 (1972)

4. Chaturani, P, Ponnalagar Samy, R: Pulsatile flow of a Casson fluid through stenosed arteries with application to blood flow. Biorheology. 23, 499-511 (1986)

5. Scott Blair, GW, Spanner, DC: An Introduction to Biorheology. Elsevier Scientific Publishing Company, Amsterdam (1974)

6. Siddiqui, SU, Verma, NK, Mishra, S, Gupta, RS: Mathematical modelling of pulsatile flow of Casson's fluid in arterial stenosis. Appl. Math. Comput. 210, 1-10 (2009)

7. Massoudi, M, Phuoc, TX: Pulsatile flow of blood using a modified second-grade fluid model. Comput. Math. Appl. 56, 199-211 (2008)

8. Mekheimer, KHS, El Kot, MA: The micropolar fluid model for blood flow through a tapered artery with a stenosis. Acta Mech Sin. 24, 637-644 (2008)

9. Mandal, PK: An unsteady analysis of non-Newtonian blood flow through tapered arteries with a stenosis. Int. J. Non-Linear Mech. 40, 151-164 (2005)

10. Varshney, G, Katiyar, VK, Kumar, S: Effect of magnetic field on the blood flow in artery having multiple stenosis: a numerical study. Int. J. Eng. Sci. Tech. 2, 67-82 (2010)

11. Nadeem, S, Sher, Akbar, N, Hayat, T, Hendi, AA: Power law fluid model for blood flow through a tapered artery with a stenosis. Appl. Math. Comput. 217, 7108-7116 (2011)

12. Nadeem, S, Sher Akbar, N: Jeffrey fluid model for blood flow through a tapered artery with a stenosis. J. Mech. Med. Biol. 11, 529-545 (2011)

13. Mustafa, N, Mandal, PK, Abdullah, I, Amin, NS, Hayat, T: Numerical Simulation of generalized Newtonian blood flow past a couple of irregular arterial stenosis. Numer. Meth. Partial Diff. Eqs. 27, 960-981 (2011)

14. Abdullah, I, Amin, NS, Hayat, T: Magnetohydrodynamic effects on blood flow through an irregular stenosis. Int. J. Number. Method Fluids. doi:10.1002/fld.2436

15. Choi, SUS: Enhancing thermal conductivity of fluids with nanoparticles. In Siginer, DA, Wang, HP (eds) Developments and Applications of Non-Newtonian Flows, vol. 66, pp. 99-105, ASME, New York (1995)

16. Nadeem, S, Lee, C: Boundary layer flow of nanofluid over an exponentially stretching surface. Nanoscale Res. Lett. 7, 94 (2012)

17. Khan, WA, Pop, I: Boundary-layer flow of a nanofluid past a stretching sheet. Int. J. Heat Mass Transfer. 53, 2477-2483 (2010)

18. Sher Akbar, N, Nadeem, S: Endoscopic effects on the peristaltic flow of a nanofluid. Commun. Theor. Phys. 56, 761-768 (2011)

19. Sher Akbar, N, Nadeem, S, Hayat, T, Hendi, AA: Peristaltic flow of a nanofluid in a non-uniform tube. Heat Mass Transfer. 48, 451-459 (2012)
20. Sher Akbar, N, Nadeem, S, Hayat, T, Hendi, AA: Peristaltic flow of a nanofluid with slip effects. Meccanica. 5, 1283-1294 (2012)

21. He, JH: Homotopy perturbation technique, a new nonlinear analytical technique. Comput. Methods. Appl. 135, 73-79 (2003)

22. $\mathrm{He}, \mathrm{JH}$ : Application of homotopy perturbation method to nonlinear wave equations. Chaos, Solitons Fractals. 26, 695-700 (2005)

doi:10.1186/2228-5326-3-35

Cite this article as: Nadeem et al: Nanoparticle analysis for blood flow of Prandtl fluid model with stenosis. International Nano Letters 2013 3:35.

\section{Submit your manuscript to a SpringerOpen ${ }^{\circ}$ journal and benefit from:}

- Convenient online submission

Rigorous peer review

- Immediate publication on acceptance

- Open access: articles freely available online

- High visibility within the field

- Retaining the copyright to your article

Submit your next manuscript at $>$ springeropen.com 